\title{
A Bayesian Inference Approach for Bivariate Weibull Distributions Derived from Roy and Morgenstern Methods
}

\author{
Ricardo Puziol de Oliveira ${ }^{1, *}$, Marcos Vinícius de Oliveira Peres ${ }^{2}$, Milene Regina dos Santos ${ }^{2}$, \\ Edson Zangiacomi Martinez ${ }^{2}$, Jorge Aberto Achcar ${ }^{2}$ \\ ${ }^{1}$ Department of the Environment, State University of Maringá, Umuarama, Brazil \\ ${ }^{2}$ Department of Social Medicine, University of São Paulo, Ribeirão Preto, Brazil
}

\begin{abstract}
Bivariate lifetime distributions are of great importance in studies related to interdependent components, especially in engineering applications. In this paper, we introduce two bivariate lifetime assuming three-parameter Weibull marginal distributions. Some characteristics of the proposed distributions as the joint survival function, hazard rate function, cross factorial moment and stress-strength parameter are also derived. The inferences for the parameters or even functions of the parameters of the models are obtained under a Bayesian approach. An extensive numerical application using simulated data is carried out to evaluate the accuracy of the obtained estimators to illustrate the usefulness of the proposed methodology. To illustrate the usefulness of the proposed model, we also include an example with real data from which it is possible to see that the proposed model leads to good fits to the data.
\end{abstract}

Keywords Bayesian analysis, bivariate lifetime model, Morgenstern family, stress-strength property.

AMS 2010 subject classifications 62H10, 62N02

DOI:10.19139/soic-2310-5070-1240

\section{Introduction}

Multivariate survival data are usual in many areas of application. A special characteristic of multivariate data is the presence of a dependence structure between the random variables. This usually occurs when an individual response is reported by a vector related to the occurrence of two or more events of interest, or when different individuals have dependent event times (see [16], [17] and [18]). A popular technique to model this dependence, is the use of frailty models proposed by [64]. In these models, one or more random effects (latent non-observed variables) are included to model the dependence between the observations. As an alternative for this approach, we could assume different existing multivariate or bivariate parametric lifetime distributions introduced in the literature where some parameters are related to the dependence structure between the lifetimes, see for example, [34], [9],[26], [4], [33], [18], [3], [19] and [41].

Other possibility in the statistical data analysis of dependent lifetime data is the use of models constructed using different copula functions, described for example by [40] and [25]. Copula functions are used to build multivariate probability distributions with different dependence structures assuming specified marginal probability distributions for the random variables [56]. In the special case of only two lifetimes, bivariate models derived from copula functions have been used by many authors, see for example, [29], [35], [2], [43], [39], [52], [65] and [20].

\footnotetext{
${ }^{*}$ Correspondence to: Ricardo Puziol de Oliveira (Email: rpuziol.oliveira@ gmail.com). Department of the Environment, State University of Maringá, Umuarama, Brazil.
}

ISSN 2310-5070 (online) ISSN 2311-004X (print)

Copyright (C) 2021 International Academic Press 
Considering the special case of only two lifetimes, the literature presents many different techniques to construct a bivariate distribution as using copula functions, using trivariate reductions or specifying conditional and marginal distributions ([31]; [6]). In this paper, we consider two methods to construct new bivariate Weibull distributions with univariate marginal Weibull distributions: the use of the Morgenstern family proposed by [37] and the use of the bivariate dependence model proposed by [53]. A primary advantage of both methods is that the resulting form of the distribution function is less complex when compared to other existing methods introduced in the literature. It is important to point out that both methods can be used when we assume different marginal probability distributions for the lifetimes and we have some information on the dependence structure between the observations. The literature presents many studies on the Morgenstern type distributions (see for example [21], [24], [54], [13], [14], [60] and [43]). However, in the best of our knowledge, the literature does not introduce new bivariate distributions obtained using the Roy's method. Some details of both methods to construct a bivariate distribution are presented by [23].

Let $X$ and $Y$ be two continuous random variables having cumulative distribution functions given, respectively, by $F_{X}(x)=P(X \leq x)$ and $F_{Y}(y)=P(Y \leq y)$. Based on the Morgenstern copula function, the joint cumulative distribution function $(c d f)$ is given by,

$$
F(x, y)=F_{X}(x) F_{Y}(y)\left[1+\lambda S_{X}(x) S_{Y}(y)\right]
$$

where $S_{X}(x)=1-F_{X}(x)$ and $S_{Y}(y)=1-F_{Y}(y)$. Thus, the corresponding joint probability density function $(p d f)$ is given by,

$$
f(x, y)=f_{X}(x) f_{Y}(y)\left[1+\lambda\left(1-2 S_{X}(x)\right)\left(1-2 S_{Y}(y)\right)\right]
$$

where the parameter $\lambda$ defined in the interval $[-1,1]$ denotes the dependence parameter which indicates the degree of association between the random variables $X$ and $Y$. More details about the Morgenstern copula function can be seen in [55] and [42]. It can be seen that when $\lambda=0, X$ and $Y$ independent random variables. In addition, the corresponding joint survival function $(s f)$ is given by

$$
S(x, y)=S_{X}(x) S_{Y}(y)\left[1+\lambda F_{X}(x) F_{Y}(y)\right]
$$

Most of the properties and extensions of the Morgenstern family of distributions were studied by [5]. The conditional density function for the random variable $Y$ given $X=x$ under the Morgenstern family is defined as,

$$
f(y \mid x)=f_{Y}(y)\left[1+\lambda\left(1-2 S_{X}(x)\right)\left(1-2 S_{Y}(y)\right)\right]
$$

Alternatively, based on the Roy's bivariate dependence model for a bivariate system, the joint $s f$ and the corresponding joint $p d f$ are given respectively by,

$$
S(x, y)=S_{X}(x) S_{Y}(y) \exp \left(-\gamma H_{X}(x) H_{Y}(y)\right)
$$

and

$$
f(x, y)=S_{X}(x) S_{Y}(y) h_{X}(x) h_{Y}(y)\left[\left\{1+\gamma H_{X}(x)\right\}\left\{1+\gamma H_{Y}(y)\right\}-\gamma\right] \exp \left(-\gamma H_{X}(x) H_{Y}(y)\right)
$$

where $H_{X}(x)=-\log S_{X}(x)$ and $H_{Y}(y)=-\log S_{Y}(y)$ are the cumulative hazard functions for $X$ and $Y$, respectively. The parameter $0 \leq \gamma \leq 1$ denotes the dependence parameter that indicates the degree of dependence between the random variables $X$ and $Y$. It can be seen that when $\gamma=0, X$ and $Y$ are independent random variables. [53] presented most of the properties for this probability model. The Roys model is also regarded as the GumbelBarnett copula model [47]. The conditional density of $X$ given $Y=y$ under Roy's bivariate dependence model is defined as,

$$
f(x \mid y)=h_{X}(x) S_{X}(x)\left[\left\{1+\gamma H_{X}(x)\right\}\left\{1+\gamma H_{Y}(y)\right\}-\gamma\right] \exp \left(-\gamma H_{X}(x) H_{Y}(y)\right)
$$


Remark 1. Observe that, if the term $\exp \left(-\gamma H_{X}(x) H_{Y}(y)\right)$ is approximated in the form,

$$
\begin{aligned}
\exp \left(-\gamma H_{X}(x) H_{Y}(y)\right) & \approx \exp \left(-\gamma\left[\log \left(1-F_{X}(x)\right) \log \left(1-F_{Y}(y)\right)\right]\right) \\
& \approx\left[1+\gamma F_{X}(x) F_{Y}(y)\right]
\end{aligned}
$$

that is, with $\lambda=-\gamma$, where $0 \leq \gamma \leq 1$, we get (3) from (5). In this paper, we use both methods described above to construct a bivariate Weibull distribution. From the Weibull distribution properties, the resulting distribution may be a good alternative to data analysis with easily obtained mathematical expression for the correlation coefficient leading to a simple interpretation for the dependence structure between two random variables $X$ and $Y$. This paper is organized as follows: in Section 2, it is presented the bivariate Weibull distributions obtained under both methods and its basic properties. Some properties, the dependence structure and a mathematical expression to compute the cross-factorial moments are also provided in this section. Maximum likelihood and Bayesian estimators for the parameters of the proposed models are also presented. In Section 3 it is presented the results of an extensive numerical study using simulated data to illustrate the usefulness of the proposed methodology. Section 4 introduces a real data application of the proposed methodology. Finally, Section 5 closes the paper with some concluding remarks.

\section{The Bivariate Weibull Distribution}

Lifetime distributions have been extensively studied in the literature due to its medical and engineering applications. One such distribution which is very popular in the analysis of survival data is the Weibull distribution introduced Waloddi Weibull in 1951 (for more details, see [66]). The mathematical properties and its applicability and generalizations were studied by [15], [62], [45], [46], [67], [58], [10], [48], [38], [30], [11], [44], [49], among many others. The probability density function (pdf) of a continuous random variable $X$ with a Weibull distribution with three parameters is given by,

$$
f_{W}(x \mid \eta, \beta, \alpha)=\frac{\alpha}{\beta}\left(\frac{x-\eta}{\beta}\right)^{\alpha-1} \exp \left\{-\left(\frac{x-\eta}{\beta}\right)^{\alpha}\right\}
$$

where $x \geq \eta,-\infty<\eta<\infty$ is the location parameter where, depending on the context, can be interpreted as a delay, guarantee time, minimum lifetime, safe lifetime, shelf age, more generally it is termed origin or threshold. The parameter $\beta>0$ is the scale parameter (or characteristic life in the lifetime context) and $\alpha>0$ is the shape parameter (or Weibull-slope). A power transformation of $\mathrm{x}$ is given by,

$$
x \stackrel{\alpha, \beta, \eta}{\longrightarrow}\left(\frac{x-\eta}{\beta}\right)^{\alpha}=u^{\alpha}
$$

which helps the mathematical proof that the integral of $f_{W}(x \mid \eta, \beta, \alpha)$ with respect to $x$ is equal to 1 . The corresponding cumulative distribution function $(c d f)$ and survival function (sf) are given, respectively, by,

$$
F_{W}(x \mid \eta, \beta, \alpha)=1-\exp \left\{-\left(\frac{x-\eta}{\beta}\right)^{\alpha}\right\}
$$

and

$$
S_{W}(x \mid \eta, \beta, \alpha)=\exp \left\{-\left(\frac{x-\eta}{\beta}\right)^{\alpha}\right\}
$$

Remark 2. It is important to point out that one can replace the location parameter by the smallest order statistic, say $X_{(1)}=\min \left(X_{1}, X_{2}, \ldots, X_{n}\right)$ for a random sample of size $n$ and then consider a model $Y=X-X_{(1)}$ having location to be zero at the origin. This approach is commonly used in applications given some existing difficulties to get inferences for the location parameter. 
Usually the adequacy of a Weibull probability distribution could be verified from the following transformation of the $c d f$ given in (10):

$$
\begin{aligned}
1-F_{W}(x \mid \eta, \beta, \alpha) & =\exp \left\{-\left(\frac{x-\eta}{\beta}\right)^{\alpha}\right\} \\
\underbrace{\log \left[-\log \left(1-F_{W}(x \mid \eta, \beta, \alpha)\right)\right]}_{=: \tilde{y}} & =\alpha \underbrace{\log (x-\eta)}_{=: \tilde{x}} \underbrace{-\alpha \log (\beta)}_{=: \tilde{\beta}}
\end{aligned}
$$

that is, a linear relationship with slope $\alpha$ and intercept $\tilde{\beta}=-\alpha \log (\beta)$. The full interpretation of this linear model is presented by [49].

The hazard function $(h f)$ and cumulative hazard function $(c h f)$ for the three parameter Weibull distribution are given, respectively, by,

$$
h_{W}(x \mid \eta, \beta, \alpha)=\frac{\alpha}{\beta}\left(\frac{x-\eta}{\beta}\right)^{\alpha-1} \quad \text { and } \quad H_{W}(x \mid \eta, \beta, \alpha)=\left(\frac{x-\eta}{\beta}\right)^{\alpha}
$$

The hazard function of the Weibull distribution is monotonically increasing, decreasing or constant and does not allow to model bathtub hazard forms which is often found in practice. However, in many applications related to lifetimes of equipments or maintenance times with increasing or decreasing hazard function, the Weibull distribution is a useful alternative for lifetime modeling in engineering studies. The non-central moments for the three-parameter Weibull distribution with density (8) are given by,

$$
\mathbb{E}\left[X^{r}\right]=\sum_{j=0}^{r}\left(\begin{array}{l}
r \\
j
\end{array}\right) \eta^{j} \beta^{r-j} \Gamma\left(\frac{r-j}{\alpha}+1\right)
$$

where $\mathbb{E}[X]=\eta+\beta \Gamma(1+1 / \alpha)=\mu_{X}$ and $\operatorname{Var}[X]=\eta(\eta-1)+\beta[(2 \eta+1) \Gamma(1+1 / \alpha)+\beta \Gamma(1+2 / \alpha)]$.

It is important to point out that usually there are some difficulties to get inferences for the parameters of this model under a classical likelihood inference approach. For this model, the log-likelihood function is given by,

$$
\ell(\eta, \beta, \alpha)=n[\log (\alpha)-\alpha \log (\beta)]+(\alpha-1) \sum_{i=1}^{n} \log \left(x_{i}-\eta\right)-\sum_{i=1}^{n}\left(\frac{x_{i}-\eta}{\beta}\right)^{\alpha}
$$

which satisfies the constraints $0 \leq \eta \leq \min \left\{x_{i}\right\}, 1 \leq i \leq n ; \alpha, \beta>0$. According to [49], when $\alpha, \beta$ and $\eta$ are all unknown, the log-likelihood function is not bounded and the regularity conditions are not satisfied. However, when $\eta$ is known, the maximum likelihood estimation procedure follows the same procedure used for the two parameter Weibull distribution. Another point of interest is that if $\alpha, \beta$ and $\eta$ are all unknown but $\alpha$ is restricted to $\alpha \geq 1$, then when there is no solution for the likelihood equations, the maximum value for the log-likelihood function occurs at the border of the parameter space (for more details, see [51]), that is,

$$
\widehat{\eta}=\min \left(x_{i}\right), \widehat{\beta}=\frac{1}{n} \sum_{i=1}^{n}\left(x_{i}-\widehat{\eta}\right), \widehat{\alpha}=1
$$

\subsection{A Bivariate Weibull distribution Derived From the Morgenstern's Method}

Let $X$ and $Y$ be two random variables each one having three parameter Weibull distributions with respective parameters $\left(\eta_{1}, \beta_{1}, \alpha_{1}\right)$ and $\left(\eta_{2}, \beta_{2}, \alpha_{2}\right)$. Let $S_{X}, S_{Y}$ denote the corresponding $s f$ 's given by (11) and $f_{X}, f_{Y}$ be the corresponding $p d f$ 's given by (8). Using (3) and (2), the $s f$ and $p d f$ of the Morgenstern type bivariate Weibull 
distribution, hereafter denoted as BW-Type M, are obtained as,

$$
\begin{aligned}
S(x, y) & =\exp \left\{-\left(\frac{x-\eta_{1}}{\beta_{1}}\right)^{\alpha_{1}}-\left(\frac{y-\eta_{2}}{\beta_{2}}\right)^{\alpha_{2}}\right\} \\
& \times\left[1+\lambda\left(1-\exp \left\{-\left(\frac{x-\eta_{1}}{\beta_{1}}\right)^{\alpha_{1}}\right\}\right)\left(1-\exp \left\{-\left(\frac{y-\eta_{2}}{\beta_{2}}\right)^{\alpha_{2}}\right\}\right)\right]
\end{aligned}
$$

and

$$
\begin{aligned}
f(x, y) & =\frac{\alpha_{1} \alpha_{2}}{\beta_{1} \beta_{2}}\left(\frac{x-\eta_{1}}{\beta_{1}}\right)^{\alpha_{1}-1}\left(\frac{y-\eta_{2}}{\beta_{2}}\right)^{\alpha_{2}-1} \exp \left\{-\left(\frac{x-\eta_{1}}{\beta_{1}}\right)^{\alpha_{1}}-\left(\frac{y-\eta_{2}}{\beta_{2}}\right)^{\alpha_{2}}\right\} \\
& \times\left[1+\lambda\left(1-2 \exp \left\{-\left(\frac{x-\eta_{1}}{\beta_{1}}\right)^{\alpha_{1}}\right\}\right)\left(1-2 \exp \left\{-\left(\frac{y-\eta_{2}}{\beta_{2}}\right)^{\alpha_{2}}\right\}\right)\right]
\end{aligned}
$$

where $-\infty<\eta_{1}, \eta_{2}<\infty, \beta_{1}, \beta_{2}, \alpha_{1}, \alpha_{2}>0$ and $-1 \leq \lambda \leq 1$. Plots for the joint $p d f$ and the joint $s f$ of the BWType $\mathrm{M}$ distribution for different parameter values are illustrated in Figure 1.
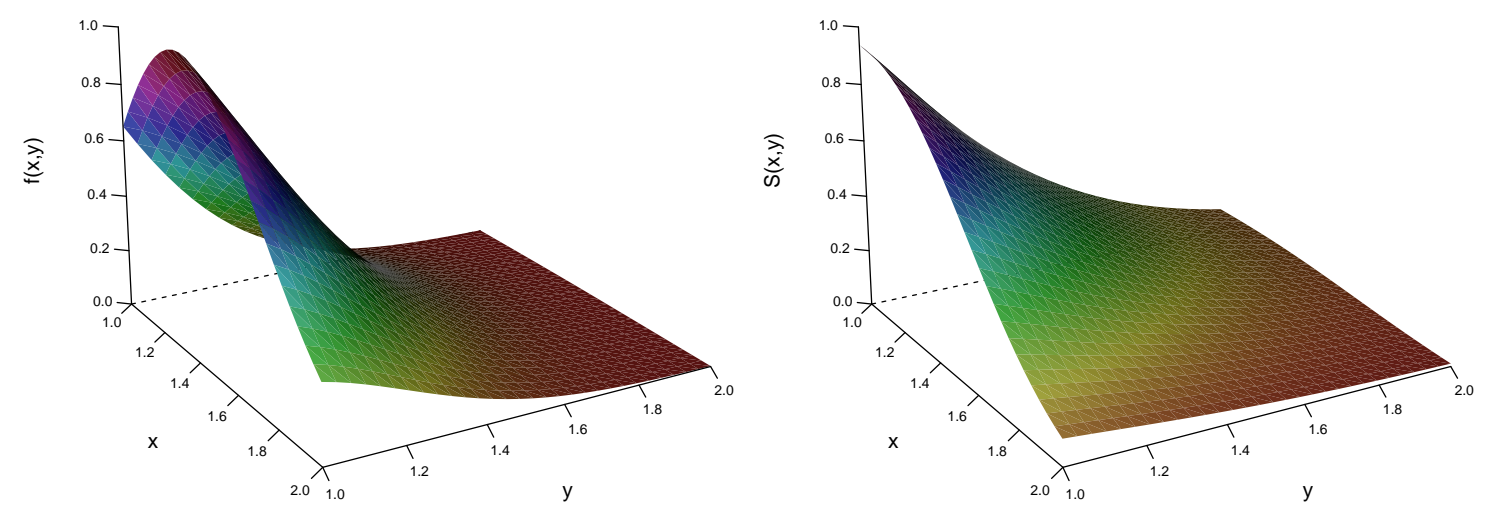

Figure 1. Behavior of the $p d f$ (left panel) and of the $s f$ (right panel) for the BW-Type M distribution assuming different parameter values.

The hazard function $(h f)$ of the BW-Type M distribution according to Basu's definition [8] is given by,

$$
h(x, y)=\frac{\alpha_{1} \alpha_{2}\left(x-\eta_{1}\right)^{\alpha_{1}-1}\left(y-\eta_{2}\right)^{\alpha_{2}-1}\left[1+\lambda\left(1-S_{w}\left(x \mid \eta_{1}, \beta_{1}, \alpha_{1}\right)\left(1-S_{w}\left(y \mid \eta_{2}, \beta_{2}, \alpha_{2}\right)\right)\right]\right.}{\beta_{1}^{\alpha_{1}} \beta_{2}^{\alpha_{2}}\left[1+\lambda\left(1-2 S_{w}\left(x \mid \eta_{1}, \beta_{1}, \alpha_{1}\right)\right)\left(1-2 S_{w}\left(y \mid \eta_{2}, \beta_{2}, \alpha_{2}\right)\right)\right]}
$$

where $S_{w}(\cdot)$ is given in (11). According to [63], a primary limitation of the definition for the hazard function defined according to Basu's definition is that this function is defined from $\mathbb{R}^{2} \rightarrow \mathbb{R}$, that is, $h(x, y)$ is not a vector quantity. To overcome this limitation, [27] defined the bivariate hazard rate function in vector form as follows,

$$
h(x, y)=\left(-\frac{\partial \log S(x, y)}{\partial x},-\frac{\partial \log S(x, y)}{\partial y}\right)
$$


Assuming the BW-Type M distribution, the vector components of the joint hazard rate function are given by,

$$
\frac{\partial \log S(x, y)}{\partial x}=\frac{\alpha_{1} \lambda\left(\frac{x-\eta_{1}}{\beta_{1}}\right)^{\alpha_{1}} S_{w}\left(x \mid \eta_{1}, \beta_{1}, \alpha_{1}\right)\left(1-S_{w}\left(y \mid \eta_{2}, \beta_{2}, \alpha_{2}\right)\right)}{\left(x-\eta_{1}\right)\left[1+\lambda\left(1-S_{w}\left(x \mid \eta_{1}, \beta_{1}, \alpha_{1}\right)\right)\left(1-S_{w}\left(y \mid \eta_{2}, \beta_{2}, \alpha_{2}\right)\right)\right]}-\frac{\alpha_{1}\left(\frac{x-\eta_{1}}{\beta_{1}}\right)^{\alpha_{1}}}{x-\eta_{1}}
$$

and

$$
\frac{\partial \log S(x, y)}{\partial y}=\frac{\alpha_{2} \lambda\left(\frac{y-\eta_{2}}{\beta_{2}}\right)^{\alpha_{2}} S_{w}\left(y \mid \eta_{2}, \beta_{2}, \alpha_{2}\right)\left(1-S_{w}\left(x \mid \eta_{1}, \beta_{1}, \alpha_{1}\right)\right)}{\left(y-\eta_{2}\right)\left[1+\lambda\left(1-S_{w}\left(x \mid \eta_{1}, \beta_{1}, \alpha_{1}\right)\right)\left(1-S_{w}\left(y \mid \eta_{2}, \beta_{2}, \alpha_{2}\right)\right)\right]}-\frac{\alpha_{2}\left(\frac{y-\eta_{2}}{\beta_{2}}\right)^{\alpha_{2}}}{x-\eta_{1}}
$$

Let $(X, Y)$ be a random vector following a BW-Type $\mathrm{M}$ distribution. The conditional distribution of $\mathrm{Y}$ given $\mathrm{X}$ $=\mathrm{x}$ and the cross factorial moment $\mathbb{E}[X Y]$ for the random vector $(X, Y)$ are given, respectively, by,

$$
\begin{aligned}
f(y \mid x) & =\frac{\alpha_{2}}{\beta_{2}}\left(\frac{x-\eta_{2}}{\beta_{2}}\right)^{\alpha_{2}-1} \exp \left\{-\left(\frac{y-\eta_{2}}{\beta_{2}}\right)^{\alpha_{2}}\right\} \\
& \times\left[1+\lambda\left(2 \exp \left\{-\left(\frac{x-\eta_{1}}{\beta_{1}}\right)^{\alpha_{1}}\right\}-1\right)\left(2 \exp \left\{-\left(\frac{x-\eta_{2}}{\beta_{2}}\right)^{\alpha_{2}}\right\}-1\right)\right]
\end{aligned}
$$

and,

$$
\begin{aligned}
\mathrm{E}[X Y] & =\lambda\left[\eta_{1}+\beta_{1} \Omega_{11}\left(\alpha_{1}\right)-\frac{\beta_{1} \Omega_{11}\left(\alpha_{1}\right)}{2^{1 / \alpha_{1}}}-\frac{\eta_{1}}{2}\right]\left[\eta_{2}+\beta_{2} \Omega_{21}\left(\alpha_{2}\right)-\frac{\beta_{2} \Omega_{21}\left(\alpha_{2}\right)}{2^{1 / \alpha_{2}}}-\frac{\eta_{2}}{2}\right] \\
& +\left[\eta_{1}+\beta_{1} \Omega_{11}\left(\alpha_{1}\right)\right]\left[\eta_{2}+\beta_{2} \Omega_{21}\left(\alpha_{2}\right)\right]
\end{aligned}
$$

where $\Omega_{i 1}\left(\alpha_{i}\right)=\Gamma\left(1+1 / \alpha_{i}\right)$ for $i=1,2$. It is important to point out that the mathematical results for the iterated integrals in (23) are obtained from [61], that is,

$$
\frac{\alpha}{\beta} \int_{0}^{\infty}(z+\eta)\left(\frac{z}{\beta}\right)^{\alpha-1} \exp \left\{-\left(\frac{z}{\beta}\right)^{\alpha}\right\} d x=\eta+\beta \Gamma(1+1 / \alpha)
$$

and,

$$
-\frac{\alpha}{\beta} \int_{0}^{\infty}(z+\eta)\left(\frac{z}{\beta}\right)^{\alpha-1} \exp \left\{-2\left(\frac{z}{\beta}\right)^{\alpha}\right\} d x=-\frac{\eta}{2}-\frac{\beta \Gamma(1+1 / \alpha)}{2^{1+1 / \alpha}}
$$

From (23), the covariance and the Pearson's correlation coefficient between the random variables $X$ and $Y$ are given, respectively, by

$$
\operatorname{cov}(X, Y)=\lambda\left[\left(\eta_{1}+\beta_{1} \Omega_{11}\left(\alpha_{1}\right)\right)-\frac{\beta_{1} \Omega_{11}\left(\alpha_{1}\right)}{2^{1 / \alpha_{1}}}-\frac{\eta_{1}}{2}\right]\left[\left(\eta_{2}+\beta_{2} \Omega_{21}\left(\alpha_{2}\right)\right)-\frac{\beta_{2} \Omega_{21}\left(\alpha_{2}\right)}{2^{1 / \alpha_{2}}}-\frac{\eta_{2}}{2}\right]
$$

and,

$$
\begin{aligned}
& \rho=\frac{\left[\left(\eta_{1}+\beta_{1} \Omega_{11}\left(\alpha_{1}\right)\right)-\frac{\beta_{1} \Omega_{11}\left(\alpha_{1}\right)}{2^{1 / \alpha_{1}}}-\frac{\eta_{1}}{2}\right]\left[\left(\eta_{2}+\beta_{2} \Omega_{21}\left(\alpha_{2}\right)\right)-\frac{\beta_{2} \Omega_{21}\left(\alpha_{2}\right)}{2^{1 / \alpha_{2}}}-\frac{\eta_{2}}{2}\right]}{\left\{\eta_{1}\left(\eta_{1}-1\right)+\beta_{1}\left[\left(2 \eta_{1}+1\right) \Omega_{11}\left(\alpha_{1}\right)+\beta_{1} \Omega_{12}\left(\alpha_{1}\right)\right]\right\}^{1 / 2}} \\
& \times \frac{\lambda}{\left\{\eta_{2}\left(\eta_{2}-1\right)+\beta_{2}\left[\left(2 \eta_{2}+1\right) \Omega_{21}\left(\alpha_{2}\right)+\beta_{2} \Omega_{22}\left(\alpha_{2}\right)\right]\right\}^{1 / 2}}
\end{aligned}
$$

where $\Omega_{i 2}\left(\alpha_{i}\right)=\Gamma\left(1+2 / \alpha_{i}\right), i=1,2$. 
A measure in bivariate analysis that plays an important role is the positive quadrant dependence (PQD) property. $\mathrm{PQD}$ is a form of dependence between random variables introduced by [32]. In our case, let $(X, Y)$ be a random vector following a BW-Type $\mathrm{M}$ distribution.In this case, $X$ and $Y$ are said to be PQD if $S(x, y) \geq S(x) S(y)$. In fact, observe that, if $0 \leq \lambda \leq 1$, then,

$$
\left[1+\lambda\left(1-\exp \left\{-\left(\frac{x-\eta_{1}}{\beta_{1}}\right)^{\alpha_{1}}\right\}\right)\left(1-\exp \left\{-\left(\frac{y-\eta_{2}}{\beta_{2}}\right)^{\alpha_{2}}\right\}\right)\right]>1
$$

which implies that $S(x, y) \geq S(x) S(y)$. However, if $-1 \leq \lambda \leq 0$, then,

$$
0<\left[1+\lambda\left(1-\exp \left\{-\left(\frac{x-\eta_{1}}{\beta_{1}}\right)^{\alpha_{1}}\right\}\right)\left(1-\exp \left\{-\left(\frac{y-\eta_{2}}{\beta_{2}}\right)^{\alpha_{2}}\right\}\right)\right]<1
$$

which implies that $S(x, y) \leq S(x) S(y)$. That is, the condition of PQD only holds for positive values of $\lambda$. Otherwise, the condition $S(x, y) \leq S(x) S(y)$ implies negative quadrant dependence which holds for negative values of $\lambda$.

Another measure that plays an important role in lifetime studies is the stress-strength parameter denoted by $\mathrm{R}$. In this context, $\mathrm{R}$ is considered as a measure of reliability of a system and it gives the probability of strength $(X)$ exceeding the stress $(Y)$. Assuming that the strength $(X)$ and the stress $(Y)$ are jointly distributed according to a $\mathrm{BW}$-Type $\mathrm{M}$ distribution with dependence parameter $\lambda$, $\mathrm{R}$ is obtained as,

$$
\begin{aligned}
R & =\frac{\alpha_{1} \alpha_{2}}{\beta_{1} \beta_{2}}\left\{\int_{\eta 1}^{\infty} \int_{x}^{\infty}\left(\frac{x-\eta_{1}}{\beta_{1}}\right)^{\alpha_{1}-1}\left(\frac{y-\eta_{2}}{\beta_{2}}\right)^{\alpha_{2}-1} \exp \left\{-\left(\frac{x-\eta_{1}}{\beta_{1}}\right)^{\alpha_{1}}-\left(\frac{y-\eta_{2}}{\beta_{2}}\right)^{\alpha_{2}}\right\}\right. \\
& \left.\times\left[1+\lambda\left(1-2 \exp \left\{-\left(\frac{x-\eta_{1}}{\beta_{1}}\right)^{\alpha_{1}}\right\}\right)\left(1-2 \exp \left\{-\left(\frac{y-\eta_{2}}{\beta_{2}}\right)^{\alpha_{2}}\right\}\right)\right]\right\} d y d x
\end{aligned}
$$

which has no closed form, however, it is equal to $1 / 2$ when $\alpha_{1}=\alpha_{2}, \beta_{1}=\beta_{2}$ and $\eta_{1}=\eta_{2}$.

\subsection{A Bivariate Weibull distribution Derived From the Roy's Bivariate Dependence Model}

Let $X$ and $Y$ be two random variables each one having a three parameter Weibull distribution with parameters $\left(\eta_{1}, \beta_{1}, \alpha_{1}\right)$ and $\left(\eta_{2}, \beta_{2}, \alpha_{2}\right)$. Let $S_{X}, S_{Y}$ denote the corresponding $s f$ 's given by (11) and $f_{X}, f_{Y}$ be the corresponding $p d f$ 's given by (8). Using (5) and (6), the $s f$ and $p d f$ of the Roy type bivariate Weibull distribution, hereafter denoted as BW-Type R distribution, are given, respectively, by,

$$
S(x, y)=\exp \left\{-\left(\frac{x-\eta_{1}}{\beta_{1}}\right)^{\alpha_{1}}-\left(\frac{y-\eta_{2}}{\beta_{2}}\right)^{\alpha_{2}}-\gamma\left(\frac{x-\eta_{1}}{\beta_{1}}\right)^{\alpha_{1}}\left(\frac{y-\eta_{2}}{\beta_{2}}\right)^{\alpha_{2}}\right\}
$$

and

$$
\begin{aligned}
f(x, y) & =\frac{\alpha_{1} \alpha_{2}}{\beta_{1} \beta_{2}}\left(\frac{x-\eta_{1}}{\beta_{1}}\right)^{\alpha_{1}-1}\left(\frac{y-\eta_{2}}{\beta_{2}}\right)^{\alpha_{2}-1} \exp \left\{-\left(\frac{x-\eta_{1}}{\beta_{1}}\right)^{\alpha_{1}}-\left(\frac{y-\eta_{2}}{\beta_{2}}\right)^{\alpha_{2}}\right\} \\
& \times\left[\left\{1+\gamma\left(\frac{x-\eta_{1}}{\beta_{1}}\right)^{\alpha_{1}}\right\}\left\{1+\gamma\left(\frac{y-\eta_{2}}{\beta_{2}}\right)^{\alpha_{2}}\right\}-\gamma\right] \exp \left\{-\gamma\left(\frac{x-\eta_{1}}{\beta_{1}}\right)^{\alpha_{1}}\left(\frac{y-\eta_{2}}{\beta_{2}}\right)^{\alpha_{2}}\right\}
\end{aligned}
$$

where $-\infty<\eta_{1}, \eta_{2}<\infty, \beta_{1}, \beta_{2}, \alpha_{1}, \alpha_{2}>0$ and $0 \leq \gamma \leq 1$. Plots of the joint $p d f$ and the joint $s f$ of the BW-Type $\mathrm{R}$ distribution for different parameter values are illustrated in Figure 2. 

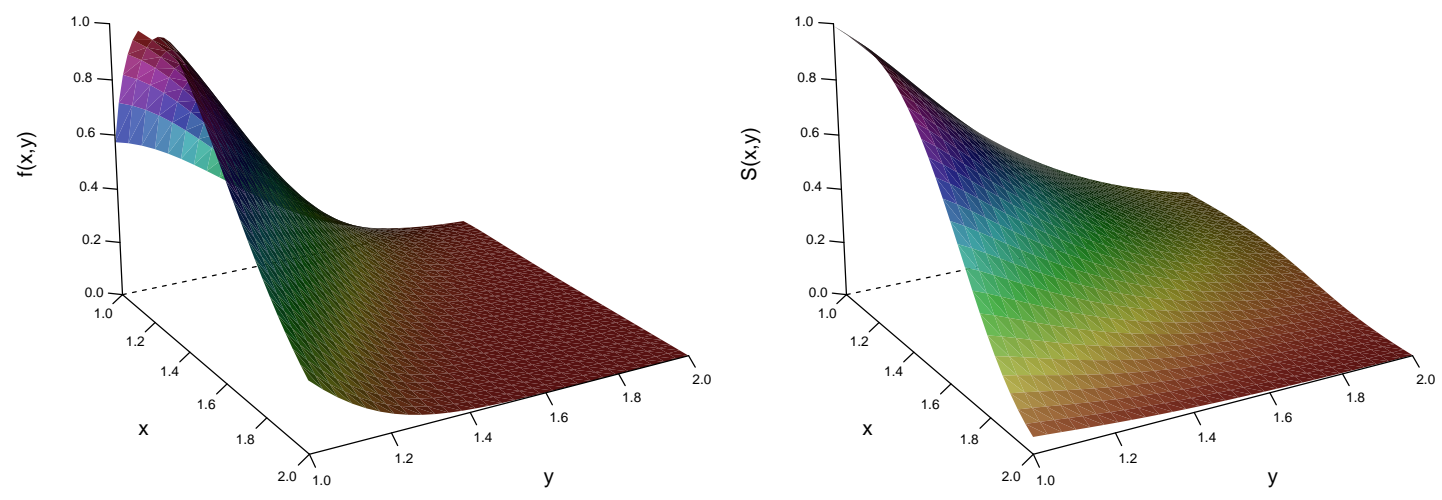

Figure 2. Behavior of the $p d f$ (left panel) and of the $s f$ (right panel) for the BW-Type R distribution assuming different parameter values.

The vector hazard function for this model is given with the components,

$$
\begin{aligned}
-\frac{\partial \log S(x, y)}{\partial x} & =\frac{\alpha_{1}\left(\frac{x-\eta_{1}}{\beta_{1}}\right)^{\alpha_{1}}\left[1+\gamma\left(\frac{y-\eta_{2}}{\beta_{2}}\right)^{\alpha_{2}}\right]}{x-\eta_{1}} \\
-\frac{\partial \log S(x, y)}{\partial y} & =\frac{\alpha_{2}\left(\frac{y-\eta_{2}}{\beta_{2}}\right)^{\alpha_{2}}\left[1+\gamma\left(\frac{x-\eta_{1}}{\beta_{1}}\right)^{\alpha_{1}}\right]}{y-\eta_{2}}
\end{aligned}
$$

which have simpler forms than the vector hazard function for the BW-Type M model. Now, let $(X, Y)$ be a random vector following a BW-Type $\mathrm{R}$ distribution. The conditional distribution of $\mathrm{Y}$ given $\mathrm{X}=\mathrm{x}$ is given by,

$$
\begin{aligned}
f(x \mid y) & =\frac{\alpha_{1}}{\beta_{1}}\left(\frac{x-\eta_{1}}{\beta_{1}}\right)^{\alpha_{1}-1} \exp \left\{-\left(\frac{x-\eta_{1}}{\beta_{1}}\right)^{\alpha_{1}}\right\}\left[\left\{1+\gamma\left(\frac{x-\eta_{1}}{\beta_{1}}\right)^{\alpha_{1}}\right\}\right. \\
& \left.\times\left\{1+\gamma\left(\frac{y-\eta_{2}}{\beta_{2}}\right)^{\alpha_{2}}\right\}-\gamma\right] \exp \left\{-\gamma\left(\frac{x-\eta_{1}}{\beta_{1}}\right)^{\alpha_{1}}\left(\frac{y-\eta_{2}}{\beta_{2}}\right)^{\alpha_{2}}\right\}
\end{aligned}
$$

An disadvantage of this model is related to the analysis of the dependence structure. For this model, the cross factorial moment $\mathbb{E}[X Y]$ as well the correlation coefficient for the random vector $(X, Y)$ has no closed form and it is needed to use numerical methods to obtain them. In the same way, there is no closed form for the stress-strength parameter although when $\alpha_{1}=\alpha_{2}, \beta_{1}=\beta_{2}$ and $\eta_{1}=\eta_{2}$, the stress-strength parameter is equal to $1 / 2$.

For positive and negative quadrant dependence, different of the BW-Type M model, only the condition $S(x, y) \leq$ $S(x) S(y)$ holds for all values of $\gamma$ assuming the BW-Type R model since $-\gamma\left(\frac{x-\eta_{1}}{\beta_{1}}\right)^{\alpha_{1}}\left(\frac{y-\eta_{2}}{\beta_{2}}\right)^{\alpha_{2}}<0$ which implies in negative quadrant dependence. 


\subsection{A Classical Approach for the Two Models}

Let $\left(x_{1}, y_{1}\right),\left(x_{2}, y_{2}\right), \ldots,\left(x_{n}, y_{n}\right)$ be a random sample of size $\mathrm{n}$ of a BW-Type $\mathrm{M}$ distribution. The contribution of the $i$ th observation to the log-likelihood function for the parameter vector $\boldsymbol{\theta}=\left(\alpha_{1}, \alpha_{2}, \beta_{1}, \beta_{2}, \eta_{1}, \eta_{2}, \lambda\right)$ is given by,

$$
\begin{aligned}
\ell(\boldsymbol{\theta}) & \propto\left(\alpha_{1}-1\right) \log \left(\frac{x_{i}-\eta_{1}}{\beta_{1}}\right)+\left(\alpha_{2}-1\right) \log \left(\frac{y_{i}-\eta_{2}}{\beta_{2}}\right) \\
& +\left\{-\left(\frac{x_{i}-\eta_{1}}{\beta_{1}}\right)^{\alpha_{1}}-\left(\frac{y_{i}-\eta_{2}}{\beta_{2}}\right)^{\alpha_{2}}\right\}+\log \left[1+\lambda\left(1-2 \exp \left\{-\left(\frac{x_{i}-\eta_{1}}{\beta_{1}}\right)^{\alpha_{1}}\right\}\right)\right. \\
& \left.\times\left(1-2 \exp \left\{-\left(\frac{y_{i}-\eta_{2}}{\beta_{2}}\right)^{\alpha_{2}}\right\}\right)\right]
\end{aligned}
$$

In the same way, the contribution of the $i$ th observation to the log-likelihood function for the vector of parameters $\boldsymbol{\theta}=\left(\alpha_{1}, \alpha_{2}, \beta_{1}, \beta_{2}, \eta_{1}, \eta_{2}, \gamma\right)$ of the BW-Type R is given by,

$$
\begin{aligned}
\ell(\boldsymbol{\theta}) & \propto\left(\alpha_{1}-1\right) \log \left(\frac{x_{i}-\eta_{1}}{\beta_{1}}\right)+\left(\alpha_{2}-1\right) \log \left(\frac{y_{i}-\eta_{2}}{\beta_{2}}\right)+\left\{-\left(\frac{x_{i}-\eta_{1}}{\beta_{1}}\right)^{\alpha_{1}}-\left(\frac{y_{i}-\eta_{2}}{\beta_{2}}\right)^{\alpha_{2}}\right\} \\
& +\log \left[\left\{1+\gamma\left(\frac{x_{i}-\eta_{1}}{\beta_{1}}\right)^{\alpha_{1}}\right\}\left\{1+\gamma\left(\frac{y_{i}-\eta_{2}}{\beta_{2}}\right)^{\alpha_{2}}\right\}-\gamma\right]+\left\{\gamma\left(\frac{x_{i}-\eta_{1}}{\beta_{1}}\right)^{\alpha_{1}}\left(\frac{y_{i}-\eta_{2}}{\beta_{2}}\right)^{\alpha_{2}}\right\}
\end{aligned}
$$

In both cases, the MLEs have no closed form, they are obtained using standard numerical optimization algorithms as the Newton-Raphson or the Nelder-Mead methods since the MLE of the parameters $\eta_{1}$ and $\eta_{2}$ depends on order statistics since $x \geq \eta_{1}$ and $y \geq \eta_{2}$. It is observed that the needed regularity conditions for the determination of the Fisher's information matrix are not verified, which is a limitation of the proposed models under a classical approach. Due to this limitation, it is considered a Bayesian approach in this study.

\subsection{A Bayesian Approach for the Two Models}

In this section we introduced a Bayesian approach for both models. Similar to what occurs in the classical maximum likelihood approach, the Bayes estimators cannot be obtained in explicit forms and it is used MCMC (Markov Chain Monte Carlo) methods to get the posterior summaries of interest. In this way, based on the observations and considering the mean square error loss function to get the Bayesian estimators, it is assumed Uniform $(0, \mathrm{k})$ (where $\mathrm{k}$ is a known constant) prior distributions for the parameters $\alpha_{i}, \beta_{i}, i=1,2, \lambda$ (BW-Type $\mathrm{M}$ model) and $\gamma\left(\mathrm{BW}\right.$-Type $\mathrm{R}$ model); an $\operatorname{Uniform}(0, \mathbf{x})$ (where $\left.\mathbf{x}=\min \left(x_{1}, \ldots, x_{n}\right)\right)$ prior distribution for the parameter $\eta_{1}$ and an Uniform $(0, \mathbf{y})$ (where $\mathbf{y}=\min \left(y_{1}, \ldots, y_{n}\right)$ ) prior distribution for the parameter $\eta_{2}$. These values for the hyperparameters were chosen in order to reflect prior knowledge of the parameters and better performance of the MCMC algorithm in terms of good convergence and computation stability. Observe that empirical Bayesian methods have been used in the elicitation of the prior distributions for the location parameters $\eta_{1}$ and $\eta_{2}$ of the proposed models (see for example, [12]). The joint posterior density function for the vector of parameters $\boldsymbol{\theta}=\left(\alpha_{1}, \alpha_{2}, \beta_{1}, \beta_{2}, \eta_{1}, \eta_{2}, \lambda\right)$ of the BW-Type $\mathrm{M}$ distribution is given by,

$$
\pi(\boldsymbol{\theta} \mid \text { data })=\frac{L(\boldsymbol{\theta}) \prod_{i=1}^{2} \pi_{i}\left(\alpha_{i}\right) \prod_{j=3}^{4} \pi_{j}\left(\beta_{j}\right) \prod_{k=5}^{6} \pi_{k}\left(\eta_{k}\right) \pi_{7}(\lambda)}{\int_{0}^{k} \int_{0}^{\mathbf{y}} \int_{0}^{\mathbf{x}} \int_{0}^{k} \int_{0}^{k} \int_{0}^{k} \int_{0}^{k} L(\boldsymbol{\theta}) \prod_{i=1}^{2} \pi_{i}\left(\alpha_{i}\right) d \alpha_{i} \prod_{j=3}^{4} \pi_{j}\left(\beta_{j}\right) d \beta_{j} \prod_{k=5}^{6} \pi_{k}\left(\eta_{k}\right) d \eta_{k} \pi_{7}(\lambda) d \lambda}
$$

Assuming the BW-Type R distribution, the joint posterior distribution of interest is obtained in the same way. Therefore, the Bayes estimator of any function of $\boldsymbol{\theta}=\left(\alpha_{1}, \alpha_{2}, \beta_{1}, \beta_{2}, \eta_{1}, \eta_{2}, \lambda\right)$, say $\omega(\boldsymbol{\theta})$, assuming the squared error loss function is given by, 


$$
\widehat{\omega}_{B}=\frac{\int_{0}^{k} \int_{0}^{\mathbf{y}} \int_{0}^{\mathbf{x}} \int_{0}^{k} \int_{0}^{k} \int_{0}^{k} \int_{0}^{k} \omega(\boldsymbol{\theta}) L(\boldsymbol{\theta}) \prod_{i=1}^{2} \pi_{i}\left(\alpha_{i}\right) d \alpha_{i} \prod_{j=3}^{4} \pi_{j}\left(\beta_{j}\right) d \beta_{j} \prod_{k=5}^{6} \pi_{k}\left(\eta_{k}\right) d \eta_{k} \pi_{7}(\lambda) d \lambda}{\int_{0}^{k} \int_{0}^{\mathbf{y}} \int_{0}^{\mathbf{x}} \int_{0}^{k} \int_{0}^{k} \int_{0}^{k} \int_{0}^{k} L(\boldsymbol{\theta}) \prod_{i=1}^{2} \pi_{i}\left(\alpha_{i}\right) d \alpha_{i} \prod_{j=3}^{4} \pi_{j}\left(\beta_{j}\right) d \beta_{j} \prod_{k=5}^{6} \pi_{k}\left(\eta_{k}\right) d \eta_{k} \pi_{7}(\lambda) d \lambda}
$$

The posterior summaries of interest are computed using the package R2jags [59] from R software considering a "burn-in sample" of size 1,000 to eliminate the effect of the initial values and a final Gibbs sample of size 2,000 taking every 10th sample from 20,000 simulated Gibbs samples. Furthermore, the convergence of the Gibbs Sampling algorithm was monitored using standard graphical methods as the trace plots of the simulated samples.

For comparison between the Bayesian models we considered the deviation information criterion (DIC) proposed by [57]. The DIC value is given by

$$
\mathrm{DIC}=D(\hat{\theta})+2 n_{p}=2 \bar{D}-D(\hat{\theta})
$$

where $D(\hat{\theta})$ is the deviance evaluated in the posterior mean of the parameter of interest obtained using MCMC simulation methods and $n_{p}$ is the effective number of parameters in the model, with $n_{p}=\bar{D}-D(\hat{\theta})$, where $\bar{D}=E[D(\theta)]$ is the posterior mean of the deviance. Lower values of DIC indicate better model fit.

\section{A Simulation Study}

In this section, it is presented an extensive numerical experiment considering various different scenarios of simulated datasets assuming different sample sizes to evaluate the performance of the Bayesian estimators of the proposed models introduced in Section 2. Moreover, to show the performance of the obtained Bayesian estimators we generated data sets with sample sizes equal to $n=20,50,100,150,300$ and fixed values for the parameters $\alpha_{1}, \alpha_{2}, \beta_{1}, \beta_{2}, \eta_{1}, \eta_{2}, \lambda$ (BW-Type $\left.\mathrm{M}\right), \gamma$ (BW-Type R) for different scenarios. The considered scenarios are presented in Table 1 .

Table 1. Fixed parameter values for each considered scenario to generate the simulated datasets for each sample size.

\begin{tabular}{ccccccccc}
\hline Scenario & $\alpha_{1}$ & $\alpha_{2}$ & $\beta_{1}$ & $\beta_{2}$ & $\eta_{1}$ & $\eta_{2}$ & $\lambda$ (BW-Type M) & $\gamma$ (BW-Type R) \\
\hline 1 & 1.50 & 1.50 & 1.20 & 1.20 & 2.00 & 2.00 & -0.20 & 0.20 \\
2 & 1.50 & 1.50 & 1.20 & 1.20 & 2.00 & 2.00 & -0.60 & 0.60 \\
3 & 1.50 & 1.50 & 1.20 & 1.20 & 2.00 & 2.00 & -0.95 & 0.95 \\
4 & 0.75 & 0.75 & 1.15 & 1.15 & 0.50 & 0.50 & 0.20 & 0.20 \\
5 & 0.75 & 0.75 & 1.15 & 1.15 & 0.50 & 0.50 & 0.60 & 0.60 \\
6 & 0.75 & 0.75 & 1.15 & 1.15 & 0.50 & 0.50 & 0.95 & 0.95 \\
\hline
\end{tabular}

The data sets were generated from a BW-Type $\mathrm{M}$ distribution using the generation algorithm of the random values for the random vector $(X, Y)$, given in the following steps:

- Step 1. Generate a random sample from $u_{1} \sim U(0,1)$ and put this value as $u_{1}=F_{W}\left(x \mid \eta_{1}, \beta_{1}, \alpha_{1}\right)$, given in (10), that is, $x=\beta_{1}\left(-\log \left(1-u_{1}\right)\right)^{1 / \alpha_{1}}+\eta_{1}$ to generate a random observation on $\mathrm{X}$;

- Step 2. Generate a random sample from $w \sim U(0,1)$ and put this value as $w=u_{1}\left(1+\lambda\left(1-u_{1}\right)\left(1-u_{2}\right)\right)-$ $u_{1} u_{2} \lambda\left(1-u_{2}\right)$ (this expression is the derivative of (1) with respect to $u_{1}$, when $F_{x}(x)=u_{1}$ and $\left.F_{y}(y)=u_{2}\right)$; then solve this equation in relation to $u_{1}$, generating a random observation $u_{2}$, from $u_{2} \sim U(0,1)$, where $u_{1}$ is obtained from step 1 ;

- Step 3. Considering $u_{2}$ obtained from step 2, put $u_{2}=F_{W}\left(y \mid \eta_{2}, \beta_{2}, \alpha_{2}\right)$, given in (10), that is, $y=$ $\beta_{2}\left(-\log \left(1-u_{2}\right)\right)^{1 / \alpha_{2}}+\eta_{2}$ to generate a random observation on $\mathrm{Y}$;

- Step 4. $\operatorname{Return}(X, Y)=(x, y)$. 
In the same way, the data sets were generated from a BW-Type R distribution using the generation algorithm of the random values for the random vector $(X, Y)$, according to [53], given in the following steps:

- Step 1. Generate a random sample from $u_{1} \sim U(0,1)$ and put this value as $u_{1}=\exp \left(-H_{Y}(y)\right)$, that is, $y=H_{Y}^{-1}\left(-\log \left(u_{1}\right)\right)$ to generate a random observation on $\mathrm{Y}$;

- Step 2. Generate a random sample from $u_{2} \sim U(0,1)$ and put this value as $u_{2}=(1+$ $\left.\gamma H_{X}(x)\right) \exp \left\{-H_{X}(x)\left(1-\gamma \log \left(u_{1}\right)\right)\right\}$ to generate a random observation on $\mathbf{X}$;

- Step 3. Return $(X, Y)=(x, y)$.

\subsection{Obtained results under the BW-Type R Model}

As a first statistical analysis for the simulated data sets, it is assumed the BW-Type R model. For a Bayesian approach, it is assumed $k=10$ for the uniform prior distributions $\mathrm{U}(0, \mathrm{k})$ for the parameters $\alpha_{i}, \beta_{i}, i=1,2$ and $k=1$ for the uniform prior distribution $\mathrm{U}(0, \mathrm{k})$ for the parameter $\gamma$. Since the results are quite similar among the different assumed scenarios, only the second scenario will be illustrated here.

The posterior summaries of interest for the second scenario are presented in Table 2. From the results in Table 2, we could conclude that the standard deviation values of the posterior means approaches to zero and the Bayes estimates get closer to the true parameter values when the sample size increases. Another point of interest is that the true parameter values are contained in the the $95 \%$ credible intervals for the parameters considering every sample size in each considered scenario. Moreover, Figure 3 shows the plots of the posterior distributions from where it could be seen that the Bayesian estimators under MSE risk are asymptotically unbiased and converge in distribution to the normal distribution for large sample sizes.

As an alternative to check the goodness of fit for the bivariate BW-Type R distribution, it is considered the use of marginal survival plots. If the marginal survival functions are well fitted for the data, then the bivariate BW-Type $\mathrm{R}$ distribution is adequate to the data (for more details, see [7], [50]). In this way, the plots of the empirical marginal survival functions and the Bayesian estimates for the marginal survival functions are presented in Figures 4 and 5, from where it could be seen the good fit of the BW-Type R probability distribution for the data sets.

Based on the different simulation data scenarios, the correlation coefficient and the stress-strength parameter can also be estimated. Table 3 shows the nominal correlation coefficient and the stress-strength parameter versus the Monte Carlo Bayesian estimators based on the simulated Gibbs samples of the parameters of the proposed model for the correlation coefficient and of the stress-strength parameter of the BW-Type R model under the second scenario. From the obtained results of Table 3, it could be concluded that the estimated correlation and the estimated stress parameter get closer to the nominal values when the sample size increases.

\subsection{Obtained results under the BW-Type M Model}

As a second statistical analysis of the simulated data sets, we assume the BW-Type M model. For a Bayesian approach, it is assumed $k=10$ for the uniform prior distributions for the parameters $\alpha_{i}, \beta_{i}, i=1,2$ and $k=1$ for the parameter $\gamma$. For this model, only the third scenario will be illustrated here (see the Supplementary Material Appendix for the other results).

The posterior summaries of interest for the third scenario are presented in Table 4. From the obtained results in Table 4, assuming the BW-Type M model, as it was observed for the results of the BW-Type R model in section 3.1, we observe that the standard deviation values of the posterior means approaches to zero and the Bayes estimates get closer to the true parameter values when the sample size increases. Also it is observed that the true parameter values are contained in the the $95 \%$ credible intervals for each parameter assuming different sample sizes in each considered scenario. The posterior distribution plots are presented in Figure 6. Moreover, from the plots of the empirical marginal survival functions and the Bayesian estimated survival functions assuming the proposed model for $X$ and $Y$ presented in Figures 7 and 8, we observe that the BW-Type M estimated marginal distributions show good agreement with the empirical survival plots, an indication of adequability of the proposed model for the datasets.

In the same way as considered for the BW-Type R model, Table 5 shows the nominal correlation coefficient and the stress-strength parameter versus the Monte Carlo Bayesian estimators based on the simulated Gibbs samples 

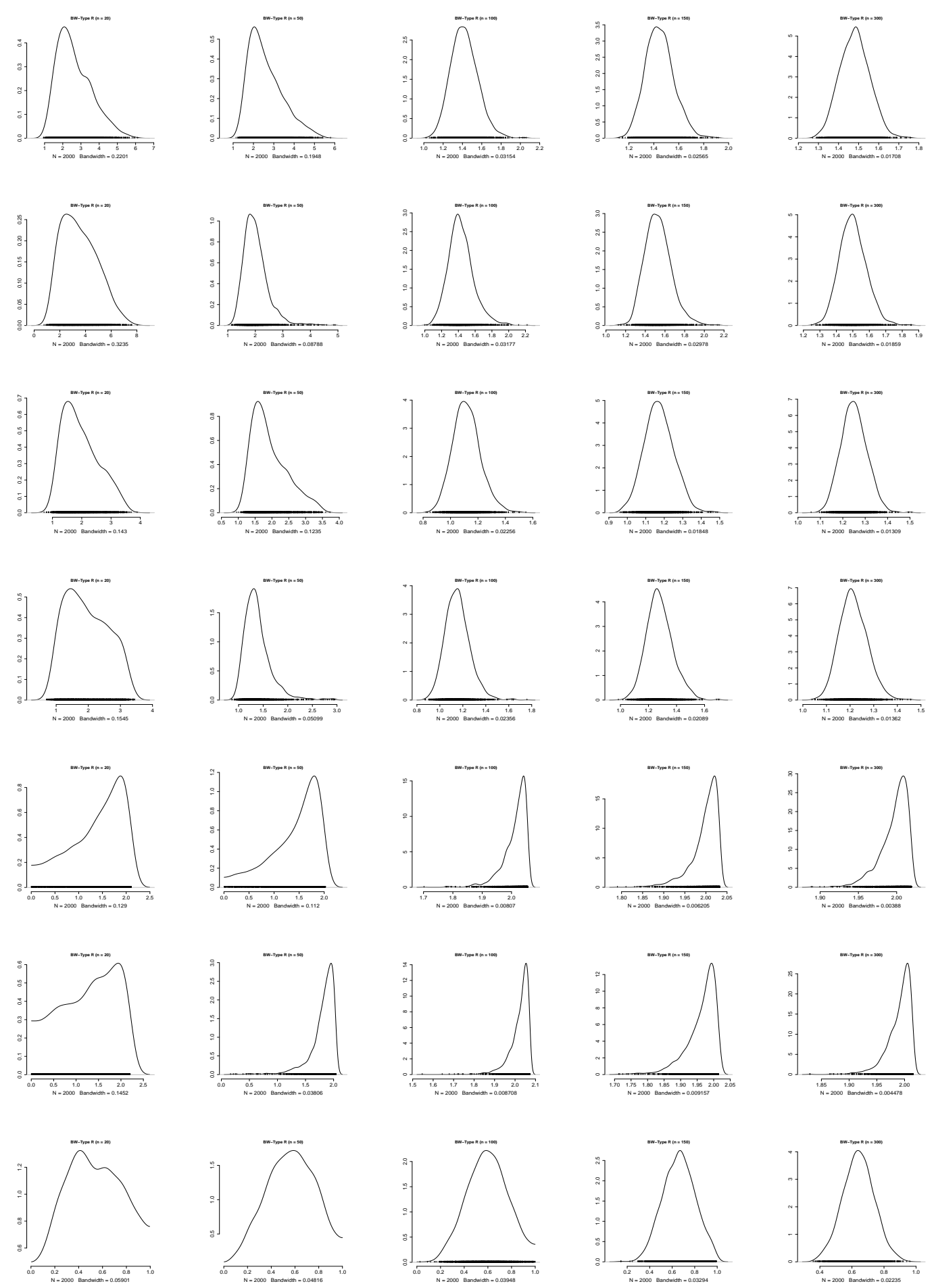

Figure 3. Posterior density plots of the Bayesian estimates $\left(\alpha_{1} \rightarrow \gamma\right)$ for each sample size considered in the Scenario 2 assuming the BW-Type R distribution. 
Table 2. Bayesian estimates for the parameters of the BW-Type R distribution considering the simulated data sets (Scenario 2).

\begin{tabular}{cccc}
\hline $\begin{array}{c}\text { Sample } \\
\text { Size }\end{array}$ & Par. & Post. Mean (Std. Dev.) & 95\% Cred. Int. \\
\hline \multirow{6}{*}{20} & $\alpha_{1}$ & $2.6497(0.9494)$ & $(1.2812,4.8540)$ \\
& $\alpha_{2}$ & $3.5356(1.3955)$ & $(1.4206,6.5256)$ \\
& $\beta_{1}$ & $1.9681(0.6169)$ & $(1.0623,3.2836)$ \\
& $\beta_{2}$ & $1.9621(0.6664)$ & $(0.9462,3.1866)$ \\
& $\eta_{1}$ & $1.3865(0.5564)$ & $(0.1489,2.0773)$ \\
& $\eta_{2}$ & $1.2730(0.6263)$ & $(0.0830,2.1614)$ \\
& $\gamma$ & $0.5200(0.2546)$ & $(0.0516,0.9638)$ \\
\hline & $\alpha_{1}$ & $2.6152(0.8403)$ & $(1.4745,4.6584)$ \\
& $\alpha_{2}$ & $2.0180(0.4597)$ & $(1.3671,3.1067)$ \\
& $\beta_{1}$ & $1.9355(0.5328)$ & $(1.2260,3.2358)$ \\
& $\beta_{2}$ & $1.3836(0.2780)$ & $(1.0197,2.0797)$ \\
& $\eta_{1}$ & $1.4244(0.4834)$ & $(0.2276,1.9841)$ \\
& $\eta_{2}$ & $1.8026(0.2374)$ & $(1.1717,2.0287)$ \\
& $\gamma$ & $0.5604(0.2078)$ & $(0.1480,0.9428)$ \\
\hline \multirow{6}{*}{100} & $\alpha_{1}$ & $1.4203(0.1394)$ & $(1.1733,1.7132)$ \\
& $\alpha_{2}$ & $1.4309(0.1518)$ & $(1.1700,1.7854)$ \\
& $\beta_{1}$ & $1.1176(0.1014)$ & $(0.9378,1.3341)$ \\
& $\beta_{2}$ & $1.1535(0.1075)$ & $(0.9667,1.3856)$ \\
& $\eta_{1}$ & $2.0116(0.0410)$ & $(1.9053,2.0536)$ \\
& $\eta_{2}$ & $2.0216(0.0496)$ & $(1.8921,2.0695)$ \\
& $\gamma$ & $0.5955(0.1703)$ & $(0.2604,0.9312)$ \\
\hline & $\alpha_{1}$ & $1.4476(0.1140)$ & $(1.2459,1.6873)$ \\
& $\alpha_{2}$ & $1.5298(0.1342)$ & $(1.3025,1.8234)$ \\
& $\beta_{1}$ & $1.1721(0.0800)$ & $(1.0247,1.3303)$ \\
& $\beta_{2}$ & $1.2800(0.0939)$ & $(1.1132,1.4848)$ \\
& $\eta_{1}$ & $1.9970(0.0328)$ & $(1.9080,2.0315)$ \\
& $\eta_{2}$ & $1.9625(0.0456)$ & $(1.8473,2.0124)$ \\
& $\gamma$ & $0.6613(0.1421)$ & $(0.3885,0.9380)$ \\
\hline & $\alpha_{1}$ & $1.4809(0.0737)$ & $(1.3447,1.6262)$ \\
& $\alpha_{2}$ & $1.5007(0.0814)$ & $(1.3565,1.6656)$ \\
& $\beta_{1}$ & $1.2503(0.0576)$ & $(1.1444,1.3659)$ \\
& $\beta_{2}$ & $1.2141(0.0587)$ & $(1.1076,1.3348)$ \\
& $\eta_{1}$ & $1.9965(0.0184)$ & $(1.9498,2.0182)$ \\
& $\eta_{2}$ & $1.9900(0.0224)$ & $(1.9487,2.0195)$ \\
& $\gamma$ & $0.6405(0.0979)$ & $(0.4472,0.8367)$ \\
\hline & & & \\
\hline 6
\end{tabular}

Table 3. The estimated correlation coefficients $(\rho)$ and the estimated stress-strenght parameters (R) for each scenario for the BW-Type R model and the nominal correlation coefficient and the stress-strength parameter.

\begin{tabular}{ccccc}
\hline Sample Size & Nominal $(\rho)$ & Estimated $(\rho)$ & Nominal $(\mathrm{R})$ & Estimated $(\mathrm{R})$ \\
\hline 20 & & -0.0588 & & 0.4256 \\
50 & & -0.0439 & & 0.4090 \\
100 & -0.0208 & -0.0285 & 0.5000 & 0.5104 \\
150 & & -0.0343 & & 0.5180 \\
300 & & -0.0383 & & 0.4895 \\
\hline
\end{tabular}

for the parameters of the model for the correlation coefficient and for the stress-strength parameter assuming the BW-Type M model under the third scenario. From the obtained results of Table 5, we conclude that the estimated correlation and the estimated stress parameter are closer to the nominal values when the sample size increases. 

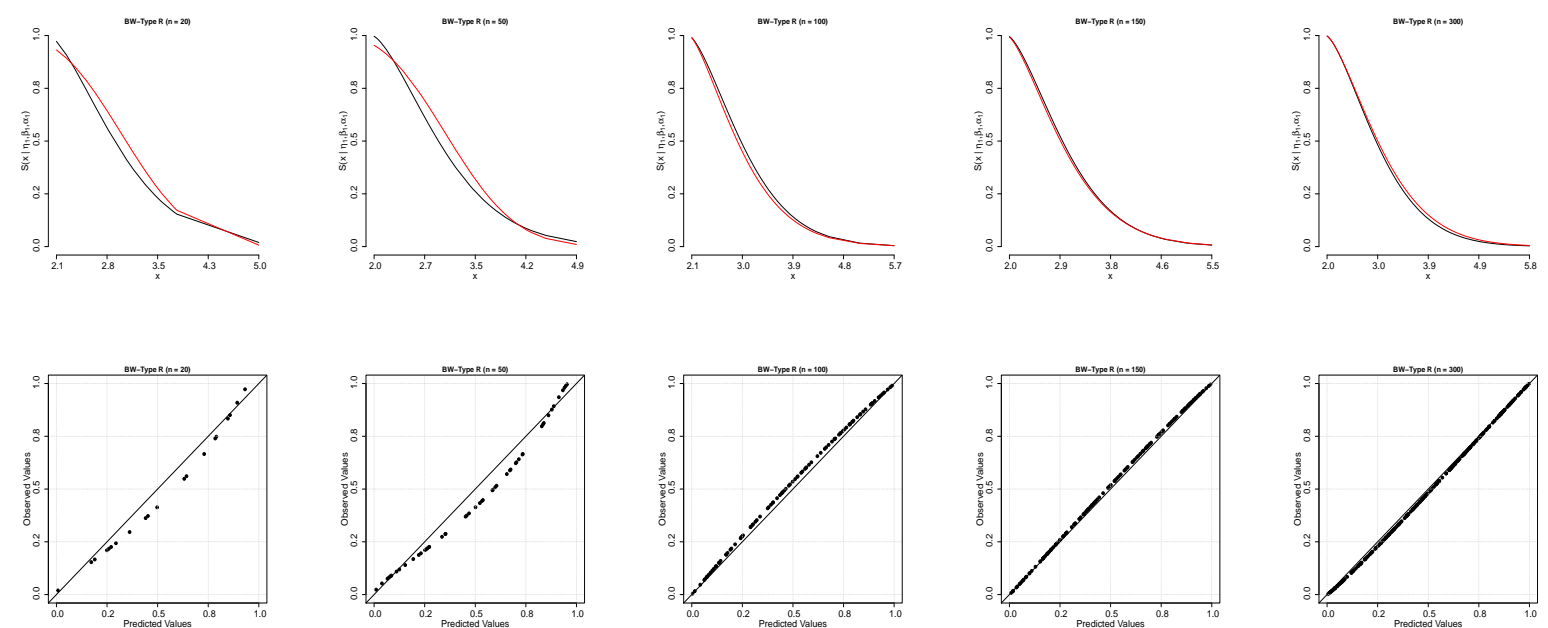

Figure 4. Plots of the empirical marginal survival function versus the Bayesian estimates for the marginal survival function for $\mathrm{X}$ assuming the BW-Type $\mathrm{R}$ distribution.
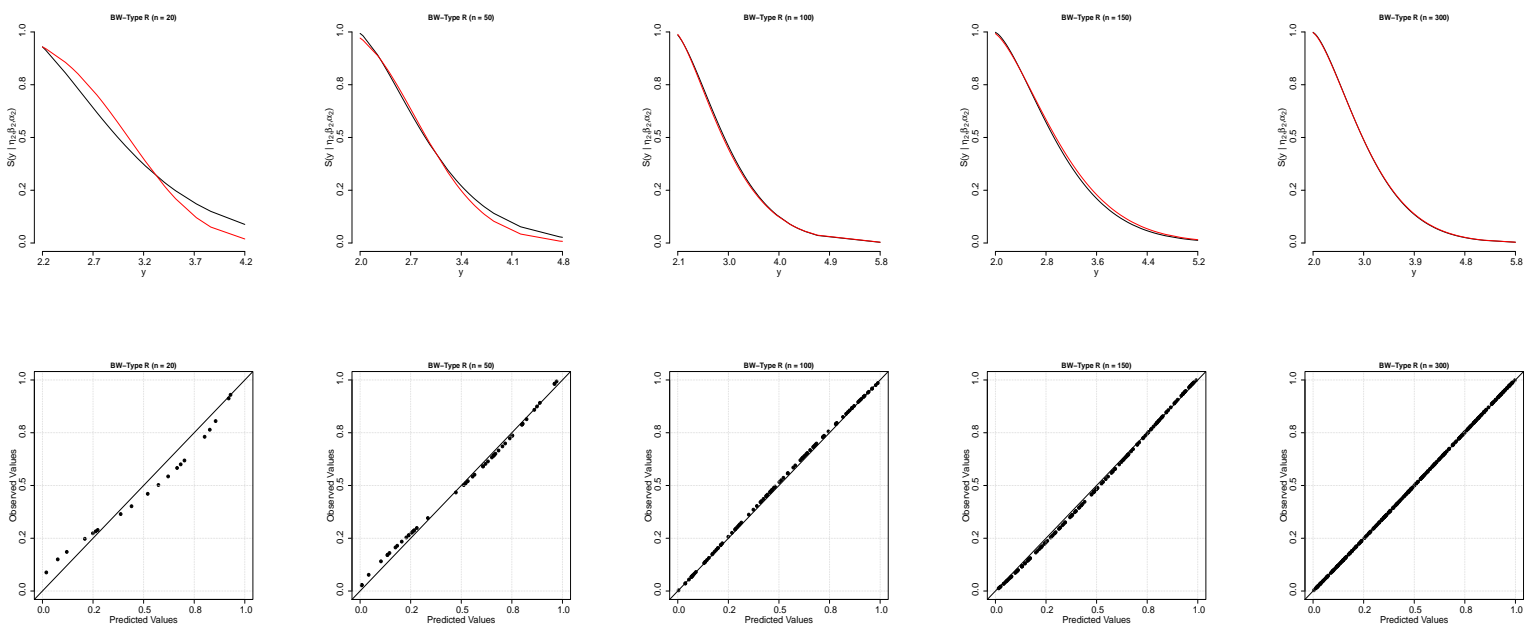

Figure 5. Plots of the empirical marginal survival function versus the Bayesian estimates for the marginal survival function for $\mathrm{Y}$ assuming the BW-Type R distribution.

\subsection{A Comparative Analysis Between Both Models}

In this subsection, we present a comparative analysis between both proposed models to evaluate the result introduced in Remark 1. For this goal, it is generated a sample of size 300 from both models assuming the first three scenarios discussed in Table 1. The same prior structures discussed previously were considered for both proposed models. The posterior summaries of interest are presented in Table 6.

From the obtained results of Table 6, we conclude that both models have approximately the same estimates for the parameters a result in agreement with Remark 1 , that is, $\exp \left(-\gamma H_{X}(x) H_{Y}(y)\right) \approx\left[1+\gamma F_{X}(x) F_{Y}(y)\right]$. In addition, for the data sets generated from the BW-Type R model, the empirical Pearson correlation coefficients for each considered scenario in Table 6 are given, respectively, by $-0.1566,-0.3806$ and -0.5149 from which it could be 

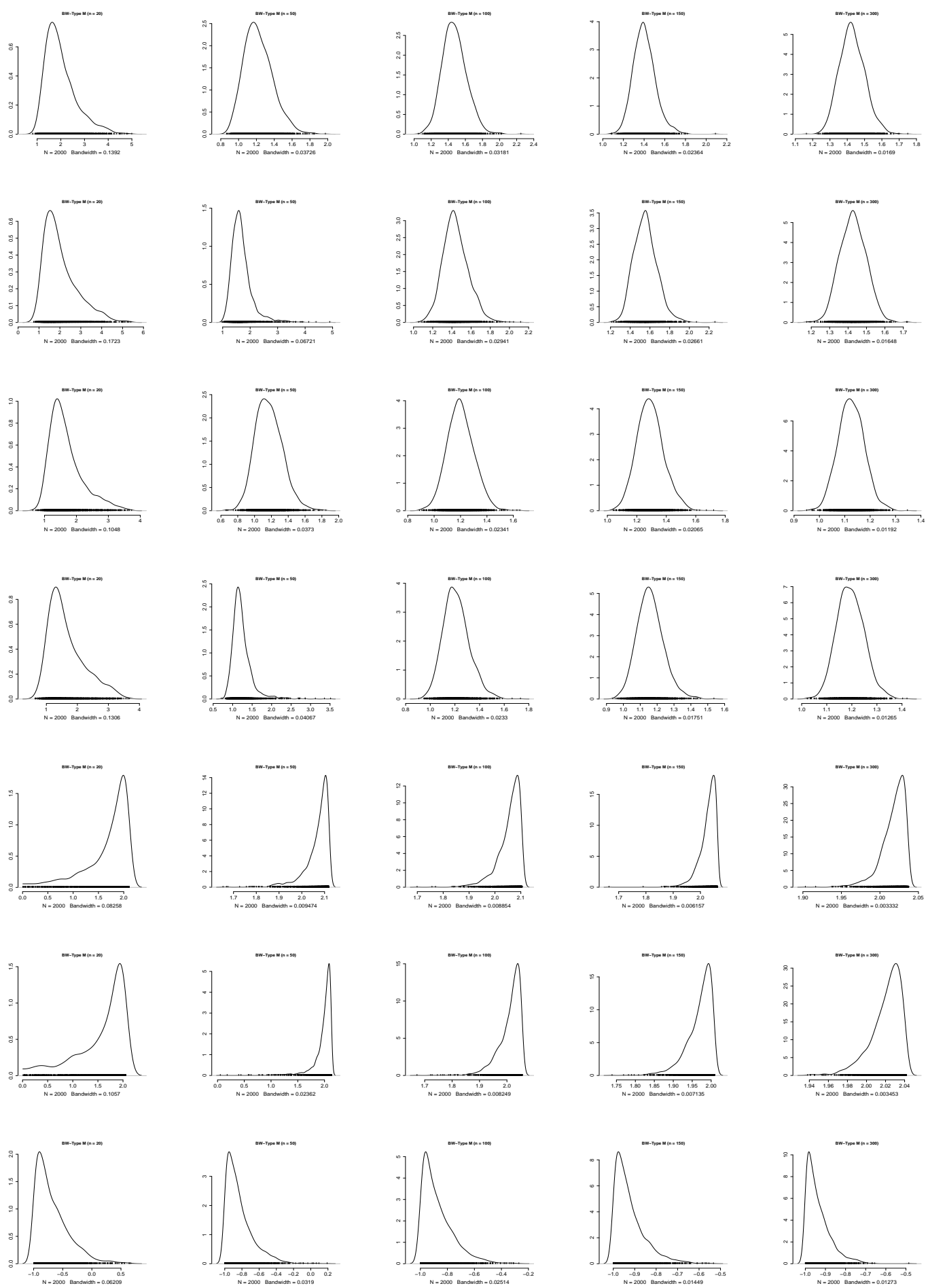

Figure 6. Posterior density plots of the Bayesian estimates $\left(\alpha_{1} \rightarrow \lambda\right)$ for each sample size considered in the Scenario 3 assuming the BW-Type M distribution. 
Table 4. Bayesian estimates for the parameters of the BW-Type M distribution considering the simulated data sets (Scenario $3)$.

\begin{tabular}{|c|c|c|c|}
\hline $\begin{array}{c}\text { Sample } \\
\text { Size }\end{array}$ & Par. & Post. Mean (Std. Dev.) & 95\% Cred. Int. \\
\hline \multirow{7}{*}{20} & $\alpha_{1}$ & $1.9959(0.6572)$ & $(1.1267,3.7241)$ \\
\hline & $\alpha_{2}$ & $2.0312(0.8067)$ & $(1.0241,4.0716)$ \\
\hline & $\beta_{1}$ & $1.6733(0.5223)$ & $(0.9910,3.0365)$ \\
\hline & $\beta_{2}$ & $1.6536(0.5850)$ & $(0.8788,3.1005)$ \\
\hline & $\eta_{1}$ & $1.6792(0.4447)$ & $(0.4253,2.0920)$ \\
\hline & $\eta_{2}$ & $1.5768(0.4971)$ & $(0.2585,2.0437)$ \\
\hline & $\lambda$ & $-0.6797(0.2860)$ & $(-0.9900,0.0434)$ \\
\hline \multirow{7}{*}{50} & $\alpha_{1}$ & $1.2230(0.1610)$ & $(0.9542,1.5796)$ \\
\hline & $\alpha_{2}$ & $1.6856(0.3896)$ & $(1.1846,2.7198)$ \\
\hline & $\beta_{1}$ & $1.1706(0.1609)$ & $(0.8952,1.5142)$ \\
\hline & $\beta_{2}$ & $1.2316(0.2591)$ & $(0.9037,1.9320)$ \\
\hline & $\eta_{1}$ & $2.0674(0.0566)$ & $(1.9023,2.1177)$ \\
\hline & $\eta_{2}$ & $1.9763(0.1999)$ & $(1.3773,2.1243)$ \\
\hline & $\lambda$ & $-0.8290(0.1600)$ & $(-0.9945,-0.4165)$ \\
\hline \multirow{7}{*}{100} & $\alpha_{1}$ & $1.4725(0.1419)$ & $(1.2172,1.7665)$ \\
\hline & $\alpha_{2}$ & $1.4398(0.1327)$ & $(1.1996,1.7166)$ \\
\hline & $\beta_{1}$ & $1.2004(0.1016)$ & $(1.0085,1.4079)$ \\
\hline & $\beta_{2}$ & $1.2113(0.1064)$ & $(1.0263,1.4427)$ \\
\hline & $\eta_{1}$ & $2.0544(0.0454)$ & $(1.9364,2.1025)$ \\
\hline & $\eta_{2}$ & $2.0094(0.0433)$ & $(1.9022,2.0538)$ \\
\hline & $\lambda$ & $-0.8709(0.1161)$ & $(-0.9953,-0.5673)$ \\
\hline \multirow{7}{*}{150} & $\alpha_{1}$ & $1.4028(0.1076)$ & $(1.2131,1.6456)$ \\
\hline & $\alpha_{2}$ & $1.5545(0.1188)$ & $(1.3531,1.8153)$ \\
\hline & $\beta_{1}$ & $1.2920(0.0916)$ & $(1.1262,1.4884)$ \\
\hline & $\beta_{2}$ & $1.1593(0.0786)$ & $(1.0192,1.3341)$ \\
\hline & $\eta_{1}$ & $2.0267(0.0318)$ & $(1.9478,2.0612)$ \\
\hline & $\eta_{2}$ & $1.9688(0.0343)$ & $(1.8809,2.0069)$ \\
\hline & $\lambda$ & $-0.9228(0.0718)$ & $(-0.9978,-0.7317)$ \\
\hline \multirow{7}{*}{300} & $\alpha_{1}$ & $1.4284(0.0729)$ & $(1.2976,1.5825)$ \\
\hline & $\alpha_{2}$ & $1.4274(0.0711)$ & $(1.2967,1.5644)$ \\
\hline & $\beta_{1}$ & $1.1252(0.0514)$ & $(1.0276,1.2282)$ \\
\hline & $\beta_{2}$ & $1.1949(0.0546)$ & $(1.0938,1.3121)$ \\
\hline & $\eta_{1}$ & $2.0173(0.0160)$ & $(1.9752,2.0357)$ \\
\hline & $\eta_{2}$ & $2.0204(0.0163)$ & $(1.9802,2.0407)$ \\
\hline & $\lambda$ & $-0.9360(0.0598)$ & $(-0.9983,-0.7731)$ \\
\hline
\end{tabular}

Table 5. The estimated correlation coefficients $(\rho)$ and the estimated stress-strenght parameters (R) for each scenario for the BW-Type M model and the nominal correlation coefficient and the stress-strength parameter.

\begin{tabular}{ccccc}
\hline Sample Size & Nominal $(\rho)$ & Estimated $(\rho)$ & Nominal $(\mathrm{R})$ & Estimated $(\mathrm{R})$ \\
\hline 20 & & -0.1977 & & 0.4560 \\
50 & & -0.1767 & & 0.5492 \\
100 & -0.2041 & -0.1892 & 0.5000 & 0.4846 \\
150 & & -0.1977 & & 0.4797 \\
300 & & -0.2073 & & 0.5279 \\
\hline
\end{tabular}

seen that using the BW-Type M model we get better estimates for the dependence parameter structures. The same happens for the data sets generated from the BW-Type M model from where the empirical Pearson correlation coefficients are given, respectively, by $-0.1229,-0.2357$ and -0.3330 .

Although under the BW-Type model the correlation is better estimated when compared to the empirical Pearson correlation coefficient, its estimated value is so far away of the nominal Pearson correlation coefficient. For a better approach in the estimation of the dependence structure, it is also considered the empirical Kendall correlation coefficient. In this case, for the data generated from the BW-Type R model, the empirical Kendall correlation 

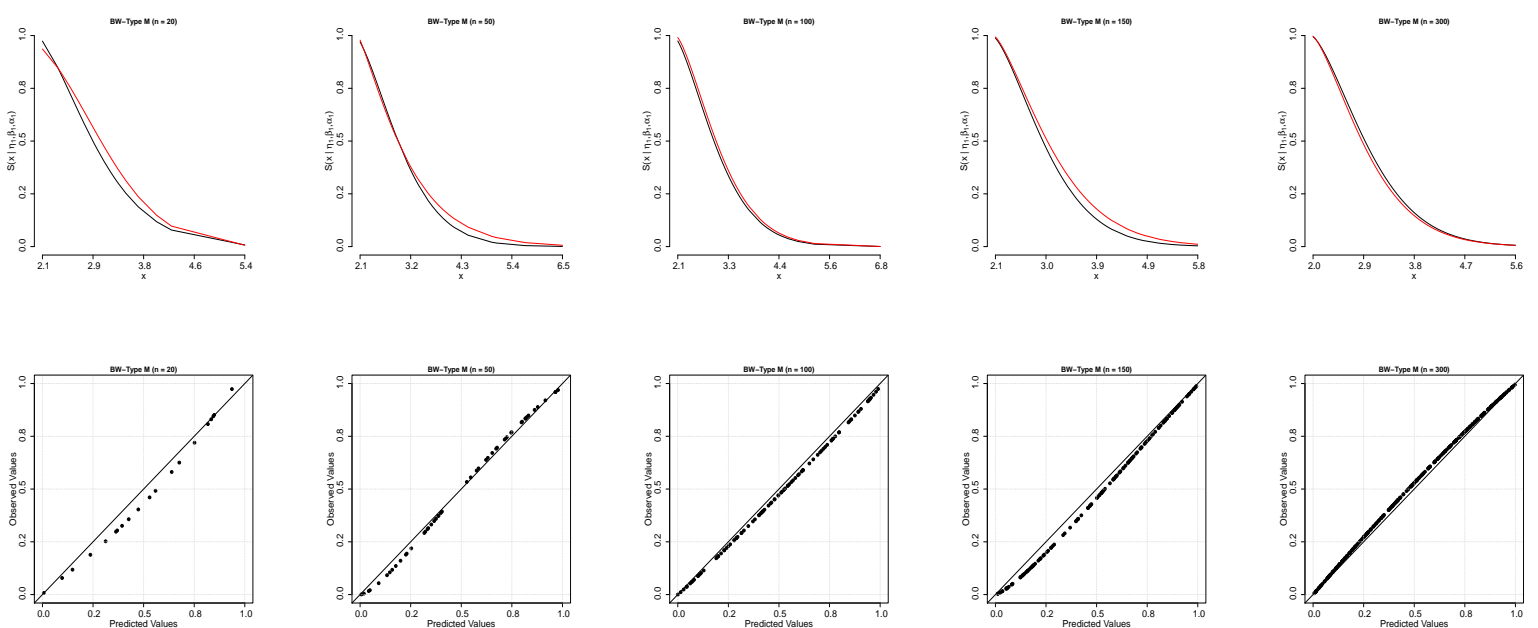

Figure 7. Plots of the empirical marginal survival function versus the Bayesian estimates for the marginal survival function for X assuming the BW-Type M distribution.
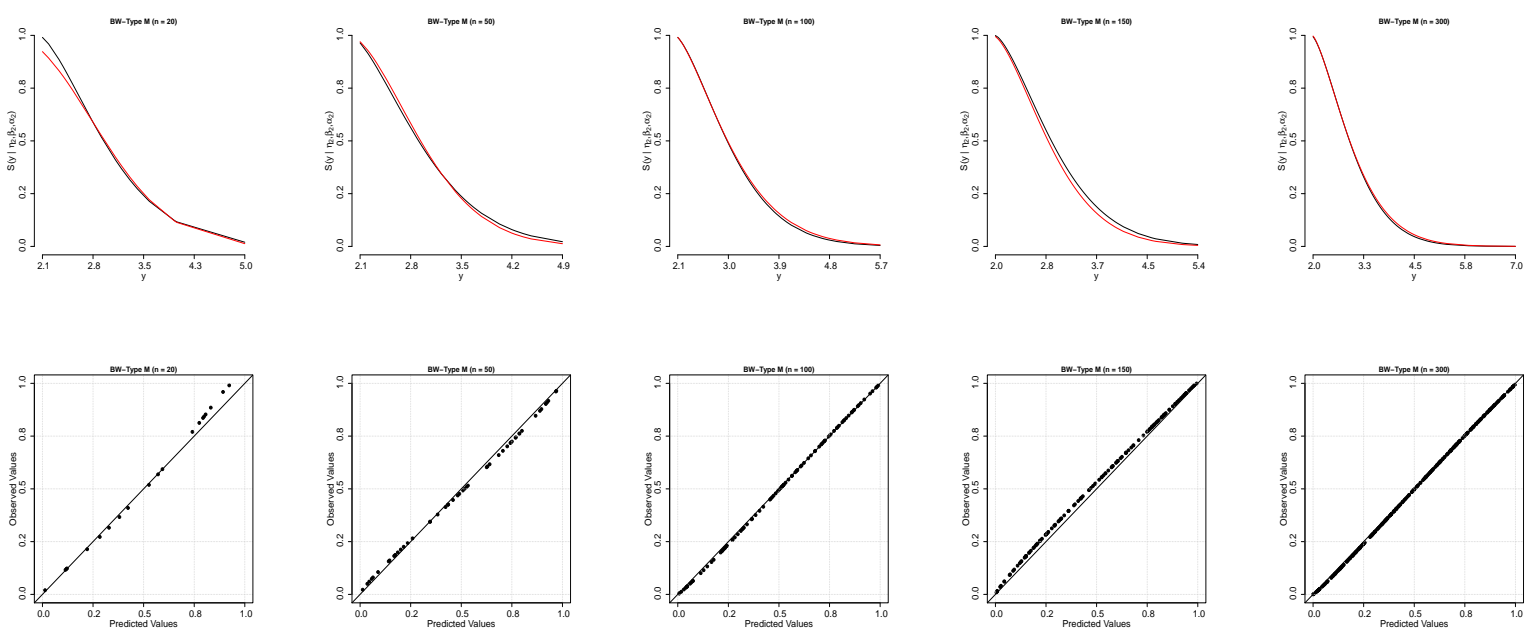

Figure 8. Plots of the empirical marginal survival function versus the Bayesian estimates for the marginal survival function for Y assuming the BW-Type M distribution.

coefficients for each considered scenario in Table 6 are given, respectively by, $-0.1001,-0.2595$ and -0.3843 from which it is observed that under the BW-Type M model we get better estimators for the dependence structure. The same happens for the data generated from the BW-Type M model from where the empirical Kendall correlation coefficients are given, respectively, by $-0.0501,-0.1348$ and -0.2726 .From these obtained results, we conclude that the BW-Type M model is the best model when compared to the BW-Type R model to deal with the dependence structure for the bivariate lifetime data. 


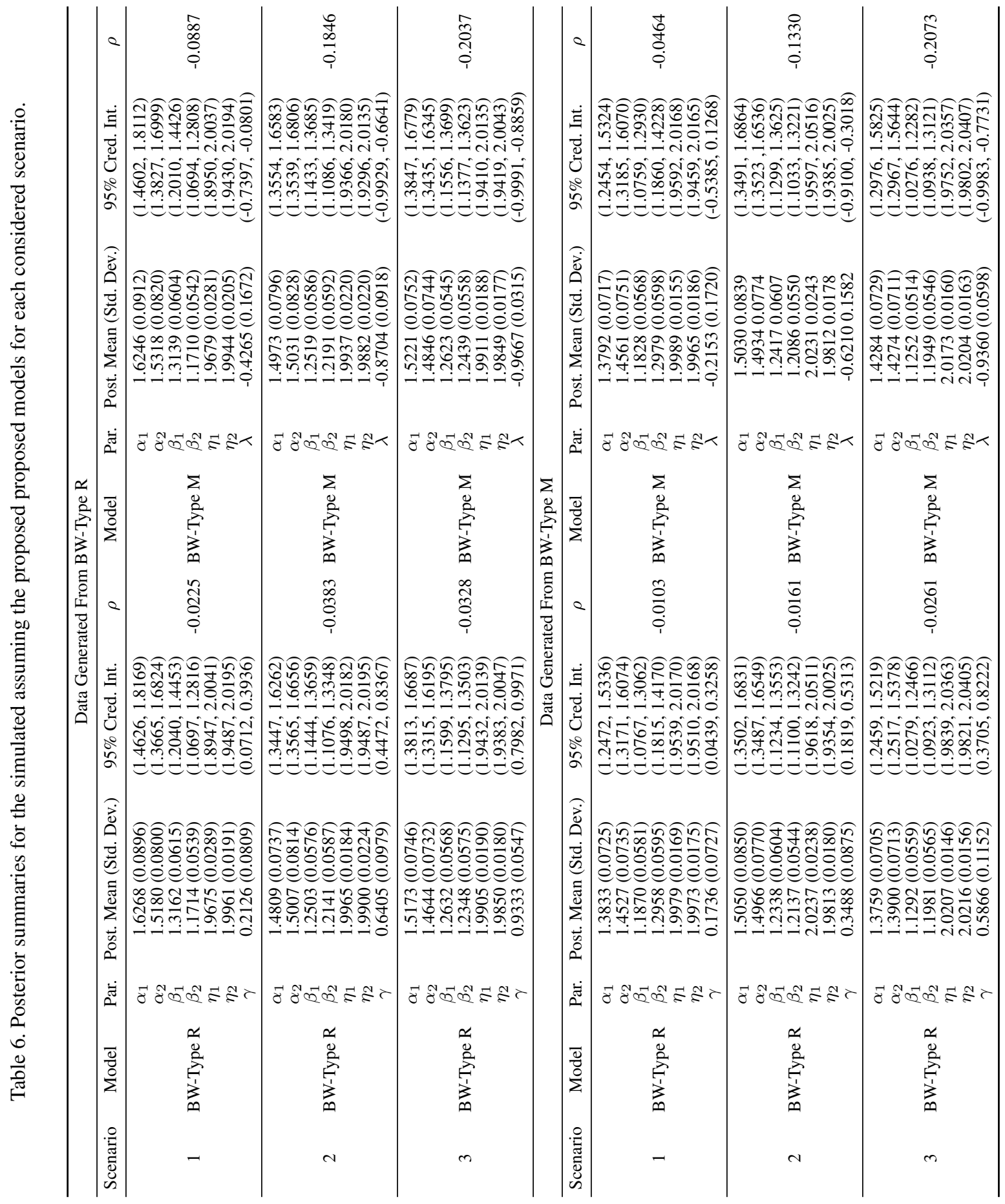


Figures 9 and 10 show the plots of the empirical marginal estimated survival functions and the Bayesian estimates of the marginal survival functions for the assumed models for the lifetimes $X$ and $Y$ from where it could be seen the similarities between both marginal survival curves for both proposed models. That is, the model derived from the Morgenstern copula survival function is approximately the same bivariate dependence model survival function obtained using the [53] approach.
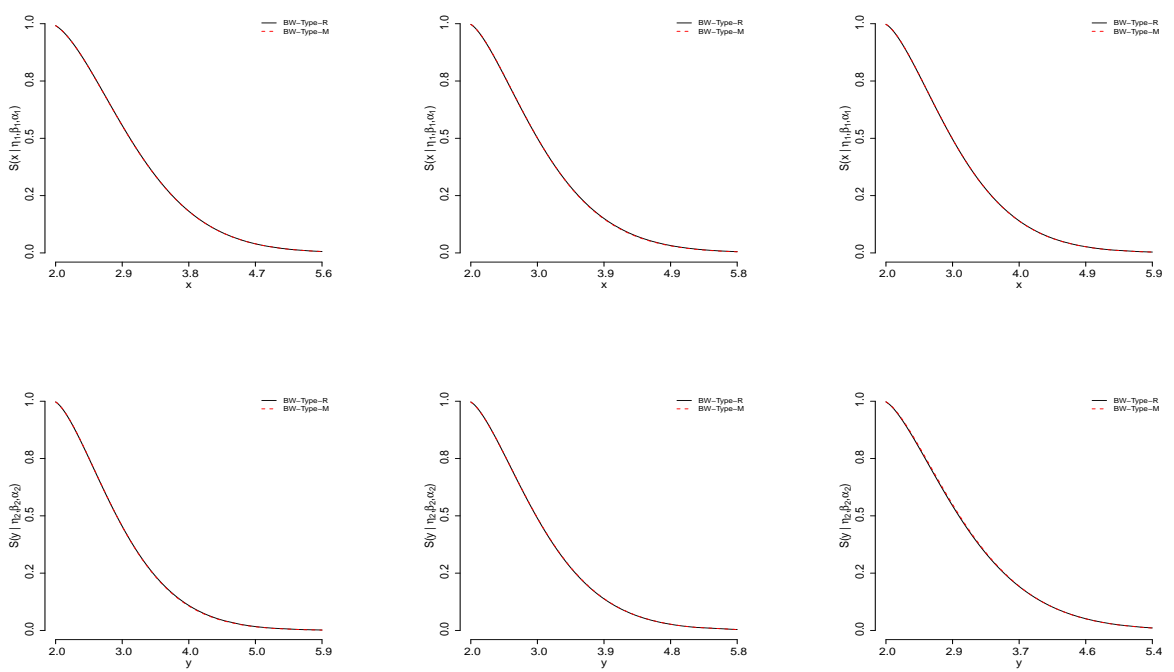

Figure 9. Plots of the marginal survival function for $\mathrm{X}$ (upper panels) and $\mathrm{Y}$ (lower panels) assuming both proposed models for the data generated from BW-Type R distribution.
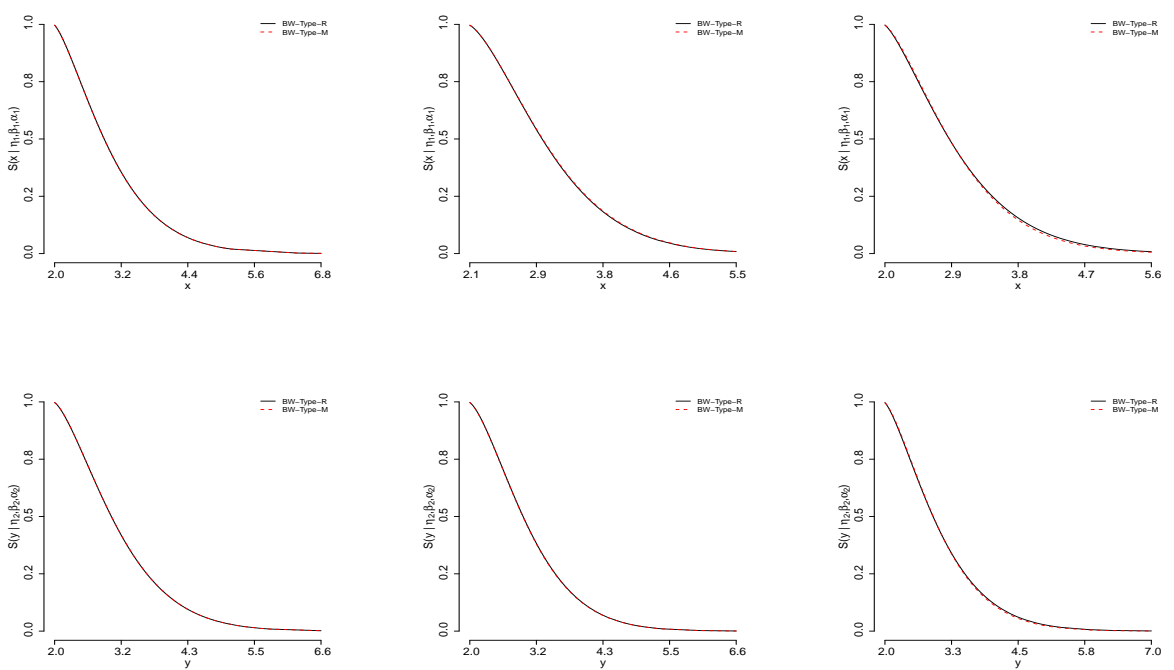

Figure 10. Plots of the marginal survival function for $\mathrm{X}$ (upper panels) and $\mathrm{Y}$ (lower panels) assuming both proposed models for the data generated from BW-Type M distribution. 


\section{A Real Data Application}

To illustrate the proposed methodology, we consider in this section, a bivariate lifetime data set introduced by [36] and also analyzed by [28]. The UEFA football data set reports: (i) there was at least one goal scored by the home team, and (ii) there was at least one goal scored by either team from the penalty spot, lack of a kick, or any other meaning unclear. Let $X$ be the time in minutes that the first goal was scored by either team and let $Y$ be the time in minutes that the first goal of any sort, was scored by the home team.

Table 7 shows the posterior Bayesian estimates for the parameters of the BW-Type M distribution and for the parameters of the BW-Type R distribution considering the UEFA football data set assuming non-informative prior distributions for the parameters of the models. Table 7 also shows the estimated values for the correlation $\rho$ between $x$ and $y$, and also the DIC value for each fitted model from where it is possible to see that the estimated values of the parameters models are very close, except for the parameter $\gamma$. From the obtained results, we observe that the estimated correlation between the times $x$ and $y$ is underestimated in the BW-Type R distribution, a possible indication that the BW-Type R model is not well fitted by the data. In fact, the DIC value suggests a better fit of the BW-Type $\mathrm{M}$ distribution for the real data.

Table 7. Posterior summaries assuming the BW Type-M and BW Type-R distributions

\begin{tabular}{cccccc}
\hline Model & Par. & Post. Mean (Std. Dev.) & $95 \%$ Cred. Int. & $\rho$ & DIC \\
\hline & $\alpha_{1}$ & $1.8464(0.2859)$ & $(1.3316,2.4194)$ & & \\
& $\alpha_{2}$ & $1.2773(0.1925)$ & $(0.9336,1.6687)$ & & \\
BW-Type M & $\beta_{1}$ & $40.8125(4.4434)$ & $(32.0396,49.4219)$ & & \\
& $\beta_{2}$ & $32.6983(4.5938)$ & $(24.4621,42.2113)$ & 0.2050 & 654.7 \\
& $\eta_{1}$ & $3.6243(1.8298)$ & $(0.2824,6.6065)$ & & \\
& $\eta_{2}$ & $1.7316(0.8160)$ & $(0.1437,2.9348)$ & & \\
& $\gamma$ & $0.6367(0.2745)$ & $(0.0020,0.9884)$ & & \\
\hline & $\alpha_{1}$ & $1.8247(0.2963)$ & $(1.2795,2.4388)$ & & \\
& $\alpha_{2}$ & $1.2367(0.1960)$ & $(0.8863,1.6529)$ & & \\
BW-Type R & $\beta_{1}$ & $41.3383(4.6089)$ & $(32.4739,50.6872)$ & & \\
& $\beta_{2}$ & $33.1537(4.8310)$ & $(24.1433,42.9413)$ & 0.0538 & 661.4 \\
& $\eta_{1}$ & $3.6580(1.8535)$ & $(0.2155,6.6663)$ & & \\
& $\eta_{2}$ & $1.7804(0.8363)$ & $(0.1319,2.9512)$ & & \\
& $\gamma$ & $0.0683(0.0685)$ & $(0.0019,0.2540)$ & & \\
\hline
\end{tabular}

Figure 11 shows the plots of the estimated BW Type-M and BW-Type-R survival functions and the nonparametric Kaplan-Meier estimates considering the UEFA football data set. From these plots there is indication of good fit of both models for the data set.

In fact for this application it is difficult to decide which assumed model is more appropriate for the data set, but there is some indication of better fit of the BW-Type M distribution considering the DIC discrimination criterion. Both models produce similar fit, however they show differences in the estimation of the dependence parameter $\gamma$.

\section{Concluding Remarks}

In this paper, it was introduced two new bivariate models obtained from marginal three parameter Weibull distributions using the Morgenstern's copula function and the bivariate dependence model introduced by [53]. For these new models, it was presented some mathematical properties and an extensive simulation study was performed to verify the effectiveness of the inferences obtained using a Bayesian approach assuming different fixed values for the parameters of the model and different sample sizes. It is important to point that the use of Bayesian methods where the posterior summaries of interest are obtained using MCMC (Markov Chain Monte Carlo) simulation techniques for bivariate lifetime distributions (see for example, [1], [22]) and the use of informative 

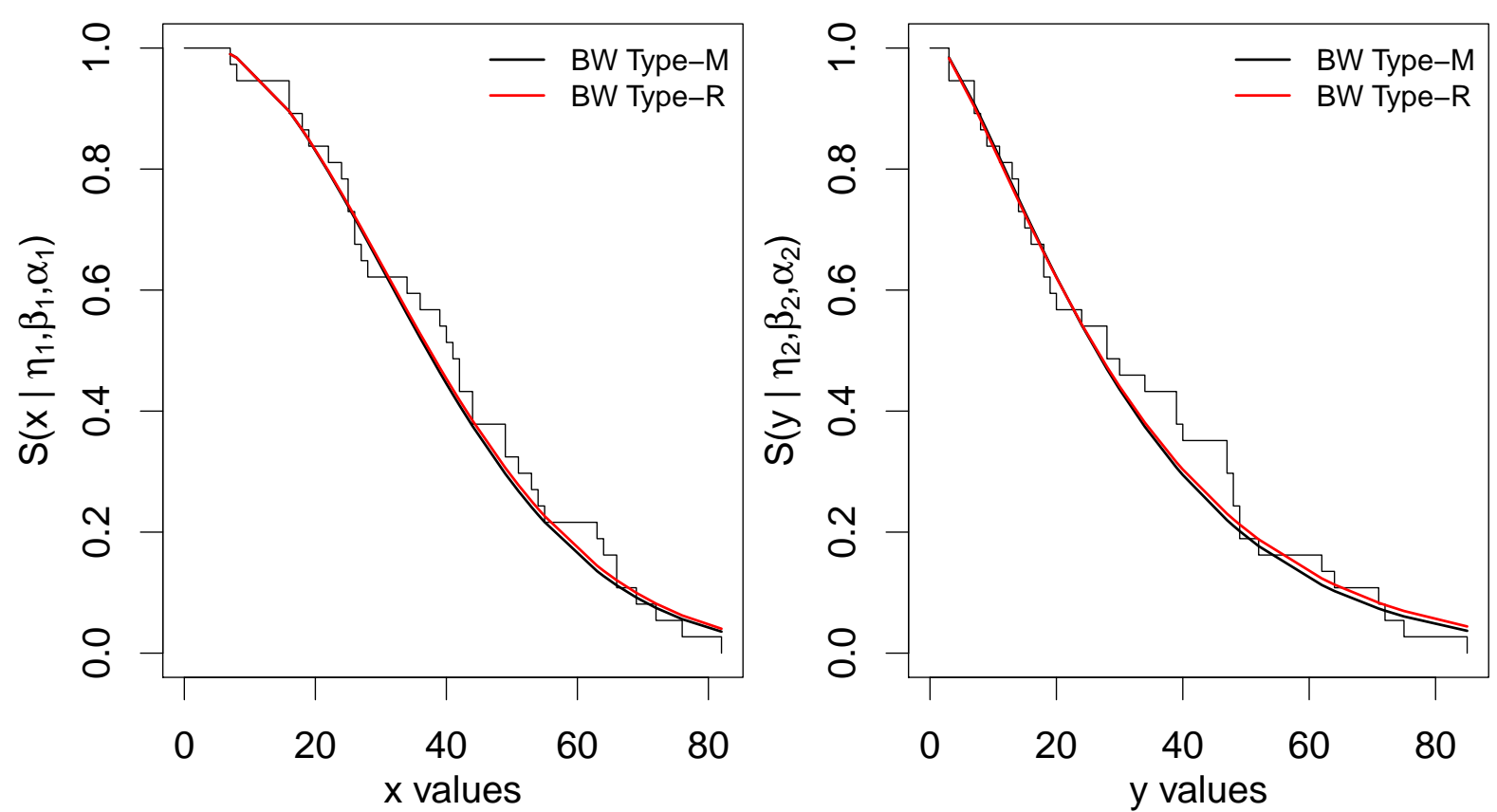

Figure 11. Plots of the marginal survival functions estimated by the Kaplan-Meier method and plots of the marginal survival functions estimated assuming the BW Type-M and BW Type-R distributions

prior distributions for the parameters $\eta_{1}$ and $\eta_{2}$ is a suitable way to get the estimators of interest, since these models do not satisfy some regularity conditions required for the use of standard asymptotic maximum likelihood inference approach. Moreover, despite the different model structure approaches considered in this study to get inferences of interest for bivariate lifetime data, it is possible to conclude that the inference results for the parameters of interest are approximately the same, however, the BW-Type R model has a lower computational cost when compared to the BW-M model, that could be an advantage for its use in lifetime data analysis.

Finally, based on the marginal survival plots in the simulation section, although the obtained results for each model are quite similar in terms of point estimators, it is possible to conclude that the use of any one of the proposed bivariate Weibull models may be a good model alternative for lifetime data analysis especially in engineering, medical or other applications of interest. In addition, the estimation of the dependence structure showed estimates close to the nominal values assuming both proposed models when the sample size increases as it was observed in the simulation study. Censoring data could also be assumed for the use of the proposed models, but this situation is outside the scope of this study and will be the subject of a future research.

Other important point that should be of interest for a future study: the use of more non-informative prior distributions for the location parameters of the three-parameter Weibull distribution, although the use of empirical Bayesian methods has been of great use in applied statistics.

\section{REFERENCES}

[1] Achcar, J. A. and Leandro, R. A. (1998). Use of Markov Chain Monte Carlo methods in a Bayesian analysis of the Block and Basu bivariate exponential distribution. Annals of the Institute of Statistical Mathematics, 50(3):403-416. 
[2] Achcar, J. A., Martinez, E. Z., and Tovar Cuevas, J. R. (2016). Bivariate lifetime modelling using copula functions in presence of mixture and non-mixture cure fraction models, censored data and covariates. Model Assisted Statistics and Applications, 11(4):261-276.

[3] Al Kadiri, M. and Migdadi, M. (2019). Estimating parameters of Morgenstern type bivariate distribution using bivariate ranked set sampling. Electronic Journal of Applied Statistical Analysis, 12(1):190-208.

[4] Arnold, B. C. and Strauss, D. (1988). Bivariate distributions with exponential conditionals. Journal of the American Statistical Association, 83(402):522-527.

[5] Balakrishnan, N. (2014). Continuous multivariate distributions. Wiley StatsRef: Statistics Reference Online.

[6] Balakrishnan, N. and Lai, C. (2009). Continuous Bivariate Distributions. Springer, New York.

[7] Balakrishnan, N. and Ristić, M. M. (2016). Multivariate families of gamma-generated distributions with finite or infinite support above or below the diagonal. Journal of Multivariate Analysis, 143:194-207.

[8] Basu, A. (1971). Bivariate failure rate. Journal of the American Statistical Association, 66(333):103-104.

[9] Block, H. W. and Basu, A. (1974). A continuous, bivariate exponential extension. Journal of the American Statistical Association, 69(348):1031-1037.

[10] Brown, W. K. and Wohletz, K. H. (1995). Derivation of the Weibull distribution based on physical principles and its connection to the Rosin-Rammler and lognormal distributions. Journal of Applied Physics, 78(4):2758 2763.

[11] Cao, Q. V. (2004). Predicting parameters of a Weibull function for modeling diameter distribution. Forest science, 50(5):682-685.

[12] Carlin, B. P. and Louis, T. A. (2010). Bayes and empirical Bayes methods for data analysis. Chapman and Hall/CRC.

[13] Chacko, M. and Thomas, P. Y. (2006). Concomitants of record values arising from Morgenstern type bivariate logistic distribution and some of their applications in parameter estimation. Metrika, 64(3):317-331.

[14] Chacko, M. and Thomas, P. Y. (2009). Estimation of parameters of Morgenstern type bivariate logistic distribution by ranked set sampling. Journal of the Indian Society of Agricultural Statistics, 63(1):77-83.

[15] Cohen, A. C. (1965). Maximum likelihood estimation in the Weibull distribution based on complete and on censored samples. Technometrics, 7(4):579-588.

[16] Crowder, M. J. (2012). Multivariate survival analysis and competing risks. Chapman and Hall/CRC.

[17] de Oliveira, R. P. and Achcar, J. A. (2018). Basu-Dhar's bivariate geometric distribution in presence of censored data and covariates: some computational aspects. Electronic Journal of Applied Statistical Analysis, 11(1):108-136.

[18] de Oliveira, R. P., Achcar, J. A., Peralta, D., and Mazucheli, J. (2019). Discrete and continuous bivariate lifetime models in presence of cure rate: a comparative study under Bayesian approach. Journal of Applied Statistics, 46(3):449-467.

[19] de Oliveira, R. P., Peres, M. V. d. O., Achcar, J. A., and Davarzani, N. (2020). Inference for the trivariate Marshall-Olkin-Weibull distribution in presence of right-censored data. Chilean Journal of Statistics, 11(2):95116.

[20] de Oliveira Peres, M. V., Achcar, J. A., and Martinez, E. Z. (2020). Bivariate lifetime models in presence of cure fraction: a comparative study with many different copula functions. Heliyon, 6(6):e03961. 
[21] D’este, G. (1981). A Morgenstern-type bivariate gamma distribution. Biometrika, 68(1):339-340.

[22] dos Santos, C. A. and Achcar, J. A. (2011). A bayesian analysis for the Block and Basu bivariate exponential distribution in the presence of covariates and censored data. Journal of Applied Statistics, 38(10):2213-2223.

[23] Emura, T., Matsui, S., and Rondeau, V. (2019). Survival Analysis with Correlated Endpoints: Joint FrailtyCopula Models. Springer.

[24] Gupta, A. K. and Wong, C. (1984). On a Morgenstern-type bivariate gamma distribution. Metrika, 31(1):327332.

[25] Joe, H. (2014). Dependence Modeling with Copulas. Chapman and Hall/CRC Press.

[26] Johnson, M. E. and Tenenbein, A. (1981). A bivariate distribution family with specified marginals. Journal of the American Statistical Association, 76(373):198-201.

[27] Johnson, N. L. and Kotz, S. (1975). A vector multivariate hazard rate. Journal of Multivariate Analysis, 5(1):53-66.

[28] Kundu, D. and Gupta, R. D. (2009). Bivariate generalized exponential distribution. Journal of multivariate analysis, 100(4):581-593.

[29] Kundu, D. and Gupta, R. D. (2011). Absolute continuous bivariate generalized exponential distribution. AStA Advances in Statistical Analysis, 95(2):169-185.

[30] Lai, C., Xie, M., and Murthy, D. (2003). A modified Weibull distribution. IEEE Transactions on reliability, 52(1):33-37.

[31] Lai, C.-D. (2006). Constructions of discrete bivariate distributions. In Advances in Distribution Theory, Order Statistics, and Inference, pages 29-58. Springer.

[32] Lehmann, E. L. (1966). Some concepts of dependence. The Annals of Mathematical Statistics, 37(5):11371153.

[33] Mahmoudi, E. and Mahmoodian, H. (2017). A new bivariate distribution obtained by compounding the bivariate normal and geometric distributions. Journal of Statistical Theory and Applications, 16(2):198-208.

[34] Marshall, A. W. and Olkin, I. (1967). A generalized bivariate exponential distribution. Journal of Applied Probability, 4(2):291-302.

[35] Martinez, E. Z. and Achcar, J. A. (2014). Bayesian bivariate generalized lindley model for survival data with a cure fraction. Computer methods and programs in biomedicine, 117(2):145-157.

[36] Meintanis, S. G. (2007). Test of fit for Marshall-Olkin distributions with applications. Journal of Statistical Planning and Inference, 137(12):3954-3963.

[37] Morgenstern, D. (1956). Einfache beispiele zweidimensionaler verteilungen. Mitteilingsblatt fur Mathematische Statistik, 8:234-235.

[38] Mudholkar, G. S., Srivastava, D. K., and Kollia, G. D. (1996). A generalization of the Weibull distribution with application to the analysis of survival data. Journal of the American Statistical Association, 91(436):15751583 .

[39] Nair, N. U., Sankaran, P., and John, P. (2018). Modelling bivariate lifetime data using copula. Metron, 76(2):133-153.

[40] Nelsen, R. B. (1999). An Introduction to Copulas. Springer. 
[41] Oliveira, R. P., Achcar, J. A., Mazucheli, J., and Bertoli, W. (2021). A new class of bivariate lindley distributions based on stress and shock models and some of their reliability properties. Reliability Engineering \& System Safety, 211:107528.

[42] Ota, S. and Kimura, M. (2021). Effective estimation algorithm for parameters of multivariate farlie-gumbelmorgenstern copula. Japanese Journal of Statistics and Data Science. doi:10.1007/s42081-021-00118-y.

[43] Peres, M. V. d. O., Achcar, J. A., and Martinez, E. Z. (2018). Bivariate modified Weibull distribution derived from Farlie-Gumbel-Morgenstern copula: a simulation study. Electronic Journal of Applied Statistical Analysis, 11(2):463-488.

[44] Pham, H. and Lai, C.-D. (2007). On recent generalizations of the Weibull distribution. IEEE transactions on reliability, 56(3):454-458.

[45] Philip, G. C. (1974). A generalized EOQ model for items with Weibull distribution deterioration. AIIE transactions, 6(2):159-162.

[46] Pinder III, J. E., Wiener, J. G., and Smith, M. H. (1978). The Weibull distribution: a new method of summarizing survivorship data. Ecology, 59(1):175-179.

[47] Portilla Yela, J. and Tovar Cuevas, J. R. (2018). Estimating the Gumbel-Barnett copula parameter of dependence. Revista Colombiana de Estadística, 41(1):53-73.

[48] Qiao, H. and Tsokos, C. P. (1995). Estimation of the three parameter Weibull probability distribution. Mathematics and Computers in Simulation, 39(1-2):173-185.

[49] Rinne, H. (2008). The Weibull distribution: a handbook. Chapman and Hall/CRC.

[50] Ristić, M. M., Popović, B. V., Zografos, K., and Balakrishnan, N. (2018). Discrimination among bivariate beta-generated distributions. Statistics, 52(2):303-320.

[51] Rockette, H., Antle, C., and Klimko, L. A. (1974). Maximum likelihood estimation with the Weibull model. Journal of the American Statistical Association, 69(345):246-249.

[52] Romeo, J. S., Meyer, R., and Gallardo, D. I. (2018). Bayesian bivariate survival analysis using the power variance function copula. Lifetime data analysis, 24(2):355-383.

[53] Roy, D. (2004). Bivariate models from univariate life distributions: a characterization cum modeling approach. Naval Research Logistics (NRL), 51(5):741-754.

[54] Scaria, J. and Nair, N. U. (1999). On concomitants of order statistics from Morgenstern family. Biometrical Journal: Journal of Mathematical Methods in Biosciences, 41(4):483-489.

[55] Shih, J.-H., Konno, Y., Chang, Y.-T., and Emura, T. (2019). Estimation of a common mean vector in bivariate meta-analysis under the fgm copula. Statistics, 53(3):673-695.

[56] Sklar, M. (1959). Fonctions de repartition an dimensions et leurs marges. Publ. inst. statist. univ. Paris, 8:229-231.

[57] Spiegelhalter, D. J., Best, N. G., Carlin, B. P., and Van Der Linde, A. (2002). Bayesian measures of model complexity and fit. Journal of the Royal Statistical Society: Series B (Statistical Methodology), 64(4):583-639.

[58] Stevens, M. and Smulders, P. (1979). The estimation of the parameters of the Weibull wind speed distribution for wind energy utilization purposes. Wind Engineering, 3(2):132-145.

[59] Su, Y.-S. and Yajima, M. (2012). R2jags: A package for running jags from r. R package version 0.03-08, URL http://CRAN. R-project. org/package $=$ R2jags. 
[60] Tahmasebi, S. and Jafari, A. A. (2012). Estimation of a scale parameter of Morgenstern type bivariate uniform distribution by ranked set sampling. Journal of Data Science, 10(1):129-141.

[61] Teimouri, M. and Gupta, A. K. (2013). On the three-parameter Weibull distribution shape parameter estimation. Journal of Data Science, 11(3):403-414.

[62] Thoman, D. R., Bain, L. J., and Antle, C. E. (1969). Inferences on the parameters of the Weibull distribution. Technometrics, 11(3):445-460.

[63] Vaidyanathan, V., Sharon Varghese, A., et al. (2016). Morgenstern type bivariate Lindley distribution. Statistics, Optimization \& Information Computing, 4(2):132-146.

[64] Vaupel, J. W., Manton, K. G., and Stallard, E. (1979). The impact of heterogeneity in individual frailty on the dynamics of mortality. Demography, 16(3):439-454.

[65] Wang, Y.-C., Emura, T., Fan, T.-H., Lo, S. M., and Wilke, R. A. (2020). Likelihood-based inference for a frailty-copula model based on competing risks failure time data. Quality and Reliability Engineering International, 36(5):1622-1638.

[66] Weibull, W. (1951). Wide applicability. Journal of Applied Mechanics, 103(730):293-297.

[67] Zanakis, S. H. (1979). A simulation study of some simple estimators for the three-parameter Weibull distribution. Journal of Statistical Computation and Simulation, 9(2):101-116.

\section{Appendices}

The following $R$ code can be used to obtain the Bayesian estimates, considering R2jags package.

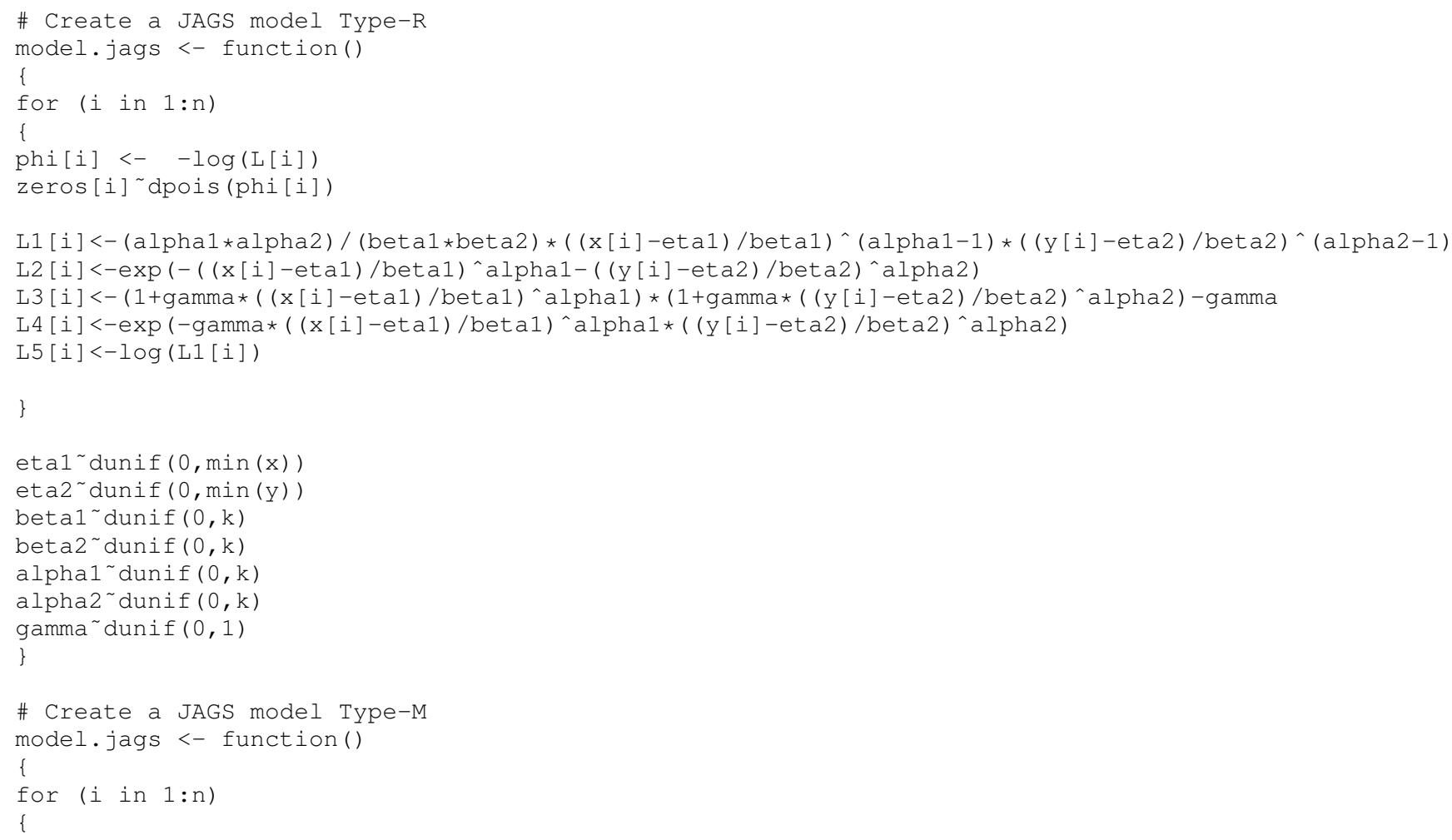




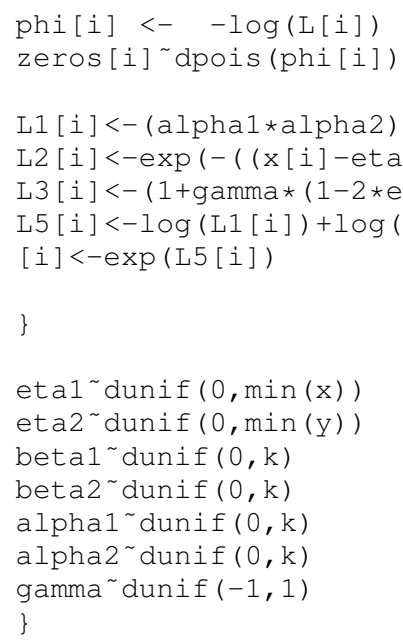

